Nuclear Instruments and Methods 175 (1980) 297-318

(c) North-Holland Publishing Company

\title{
SIMULATION OF THE MEASUREMENT BY PRIMARY CLUSTER COUNTING OF THE ENERGY LOST BY A RELATIVISTIC IONIZING PARTICLE IN ARGON
}

\author{
F. LAPIQUE and F. PIUZ \\ CERN, Geneva, Switzerland
}

Received 17 March 1980

\begin{abstract}
Energy loss of charged particles in a gas has been estimated by a computer simulation technique with a view of studying primary cluster counting as a mean of the particle identification. Primary electron yield and secondary multiplication have been estimated through a detailed calculation of the atomic processes. Results are presented for pure argon in terms of cluster size distributions and relativistic rise of the number of clusters: the latter reaches a plateau at a $\gamma$ of 200 and of level of 1.32 times minimum ionization. Limitations to detector resolution due to electronic dead time and longitudinal diffusion of the electrons drifting in the gas are presented. Such limitations are introduced in a comparison between the performance in particle separation of detectors based on energy loss, making use of pulse height measurement or cluster counting.
\end{abstract}

\section{Introduction and outline of the paper}

Most of the recent spectrometers, built or in preparation, around high energy colliders have included in their specifications a certain capability of particle identification by $\mathrm{d} E / \mathrm{d} x$ measurements. Anticipated performances are modulated by a more or less pronounced emphasis on this specific purpose with respect to the other functions the detector has to fulfil, such as position measurements, track separation and rate capability. A more specific detector, the External Particle Identifier (EPI), has been entirely designed for a $\mathrm{d} E / \mathrm{d} x$ purpose [1]. It has clearly demonstrated the precautions to be taken in order to reach an ultimate $6 \%$ fwhm energy resolution allowing a $\mathrm{p} / \mathrm{K} / \pi$ separation up to $70 \mathrm{GeV} / c$ [2]. 128 independent measurements of the ionization in $6 \mathrm{~cm}$ gas layers are required to obtain this result under real experimental conditions.

In a recent report, Walenta [3] has proposed a technique for counting the primary clusters in order to achieve particle identification over a much more restraint length of gas. The Poisson-like behaviour of this counting provides a significantly smaller fluctuation than the Landau fluctuation inherent to any pulse height measurement in the same gas layer thickness. That is responsible for this very attractive improvement.

We present in this paper a treatment of a primary cluster counting based on a computer simulation where parameters such as gas diffusion and detector time resolution can be taken into account.

We have been forced to go beyond the customary treatment of energy loss in a gas in order to meet the requirements of the cluster counter technique. We present here an improved method of energy loss calculation, which, by its generality, can be used both for cluster counting and pulse height measurements simulation. We carry on the comparison of the two methods throughout the paper, with proper comparison to pulse height measurements.

The paper is organized as follows: in sect. 2, we outline the method suggested by Walenta for counting primary clusters; in sect. 3 , we describe the principles of the simulation technique and introduce detector dead time effects and electrons diffusion in the gas; in sect. 4 , we outline the calculation of the energy loss in pure argon in terms of number of electrons locally deposited. We have grouped in appendices the details of the different steps of this calculation; in sect. 5 , we present the results of a cluster counting technique in pure argon with a realistic detector resolution and diffusion. Estimated performance of such a detector for particle separation is compared to customary $\mathrm{d} E / \mathrm{d} x$ experiments and in sect. 6, we compare our calculations with the experimental results of ref. 4 and comment about the problems arisen in gas mixtures. 


\section{Walenta's method for counting the primary clusters}

We will first recall the features of Walenta's method [3] in the general frame of the interaction of a high energy charged particle with gas atoms.

Fig. 1 shows the detector array - or Time Expansion Chamber (TEC) - used by this author. The particle traverses it along the $x$ axis, about perpendiculary to the sense wire plane.

(a) Along the trajectory, the number $N$ of primary ionizing collisions of the particle with the gas atoms fluctuates according to a Poisson law with mean value $N_{0}$ per unit length. They are spaced by distances $\Delta x_{i}$ (fig. 2) and an energy $E_{i}$ is transferred at the $i$ th collision.

(b) Each primary transfer yields electrons, depending on the $E_{i}$, on the electronic structure of the atom hit and, in first approximation, on the mean work $\bar{w}_{0}$ necessary to create an electron-ion pair in the gas mixture: a cluster is generated, of size $n_{i} \geqslant 1$. These processes will be referred to as "secondary multiplication".

(c) In the drift region $I$, the field $E_{\mathrm{d}}$ is adjusted in such a way that the drift velocity $v_{\mathrm{d}}$ of the electron is made slower than the saturated velocity $v_{\text {sat }}$ customary in a multiwire or drift chambers application, which is typically $5 \mathrm{~cm} / \mu \mathrm{s}$. Therefore time separation between clusters is increased, hence allowing cluster counting when they reach a wire in the detection chamber. We shall neglect in what follows the drift space and perturbation introduced by this chamber. Its detection efficiency is assumed to be $100 \%$. The arrival time of an electron at the boundary I-II is not determined only by $v_{\mathrm{d}}$ and the initial position $x_{i}$, but fluctuates according to the longitudinal diffusion occurring along the drift path. This fluctuation is taken to be gaussian and its standard deviation $\sigma_{i}$ can

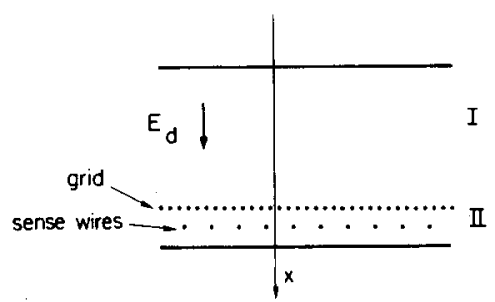

Fig. 1. Walenta's detector array. The low field drift region (I) is separated from the detection part (II) by a transparent grid (a). Particles go along the $x$ direction. In this track configuration, the detection gap has to be kept as small as possible. That minimizes the occupation time of a wire, necessary to collect the ionization deposited in (II). be written $\sigma_{0 \text { diff }} \cdot \sqrt{ } x_{i}$. We shall refer in what follows to $\sigma_{0 \text { diff }}$, which is the value of $\sigma_{i}$ taken for $x_{i}=1 \mathrm{~cm}$. The longitudinal diffusion coefficient, $\sigma_{0 \text { diff }}$, varies at low values of electrical field as $1 / \sqrt{ } E_{\mathrm{d}}$ and depends on the gas mixture [5].

(d) At the detection wire, each well separated cluster is multiplied and the induced current, processed into an electronic circuit, generates a digital signal to be counted. Let us associate to this device a dead time $\tau$ : this means, at the level of a simple counting measurement, that a sequence of several clusters, each separated by less than $\tau$, will be counted as one. By counting the number of resolved clusters, an estimate of the particle velocity is obtained.

\section{Description of the simulation technique of primary counting}

We shall describe this measurement, experimentally based on time separation, in terms of a spatial variable, the $x$ coordinate: the time resolution $\tau$ of the electronics is associated to the spatial resolution of the detector through

$\rho=v_{\mathrm{d}} \tau$.

To fix ideas, let us take for $v_{\mathrm{d}}$ the value $1 \mathrm{~cm} / \mu$ five times smaller than the velocity $v_{\text {sat }}$. If $\tau$ is taken as $10 \mathrm{~ns}$, the detector resolution will be $100 \mu \mathrm{m}$. This number may be decreased by still reducing $v_{\mathrm{d}}$ at the price of an increase of the diffusion which varies as $\sim E_{\mathrm{d}}^{-1 / 2}$.

It must be pointed out that the simple definition of $\tau$ given in sect. 2(d) can certainly be refined by using a more sophisticated electronics and performing a more detailed measurement [4] than digital counting alone. However, any experimental method will always show a certain resolution, small as it may be. It will be seen later that the best detector resolution will not necessarily provide the optimum performance. We shall keep therefore in our simulation the point of view of a "dead space" experiment, characterized by the variable $\rho$, possibly chosen from 0 to any finite value.

The simulation program consists of the following steps:

(1) Location of the primary collisions along the $x$ coordinate, from which the $x_{i}$ distribution is deduced.

(2) Energy transfer calculation for a particle of 
given Lorentz factor $\gamma$ going through the gas (argon).

(3) Secondary multiplication mechanisms which provide the Cluster Size Distribution (CSD) (the energy loss spectrum will also be obtained).

(4) The effect on the cluster counting of the diffusion during the drift and of the detector resolution, with a given CSD.

As we will see later on, the combined action $\sigma_{0 \text { diff }}$ and $\rho$ on the CSD of a real gas, will lead to a complex situation where the relative influence of these parameters may not be apparent. We shall then first detail points (1) and (4) in the frame of simpler situations such as imposed CSD or $\sigma_{0 \text { diff }}=0$. This latter case together with $\rho=0$, represents a "perfect primary counting" experiment, possibly feasible with a different collection geometry and will be taken hereafter as a reference to the "best result achievable".

The part of the program treating the primary and secondary processes of the energy deposition in the gas [(2) and (3)] are discussed in appendices B to $F$ of this paper. Their main drawbacks, CSD and $\mathrm{d} E / \mathrm{d} x$ spectrum, are discussed in sect. 4 .

\subsection{Cluster counting simulation}

A Monte Carlo technique (appendix A) gives the coordinates $x_{i}$ at which the $N$ collisions have occurred in a gas layer $l$, when an expected average has been fixed to a value $N_{0}$ (fig. 2). The distribution of the distances $\Delta x_{i}$ separating adjacent collisions is then obtained (fig. 3).

In the case of $\sigma_{0 \text { diff }}=0$, the primary clusters are considered as point like. This assumption is valid for energy transfers smaller than $1000 \mathrm{eV}$ as the range of a $1 \mathrm{keV}$ electron is of the order of $10 \mu \mathrm{m}$ in argon [6]. As seen in sect. 4.2, the fraction of transfers with energy larger than $1 \mathrm{keV}$ is small $(<1 \%)$; their production angle approaching $90^{\circ}$ with respect to the incident trajectory [7], we shall then consider the secondary electrons drifting aside parallel to this trajec-

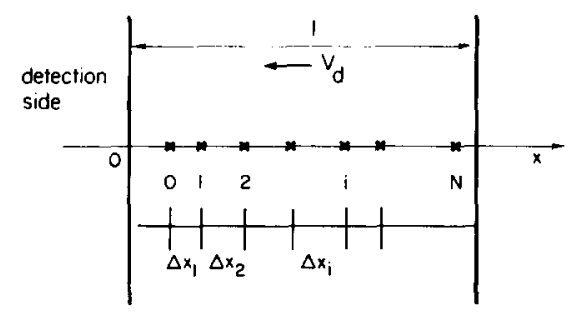

Fig. 2. Location of the primary collisions $(x)$ into the drift gap (gas layer of thickness $l$ ), at the initial time.

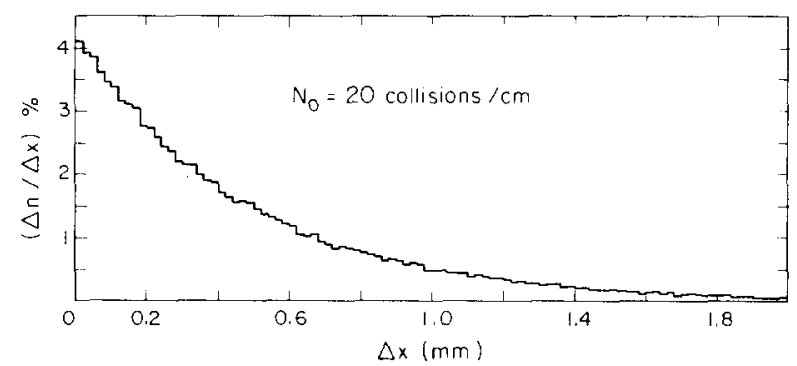

Fig. 3. Distribution of the intervals $\Delta x_{i}$ between adjacent primary clusters. In this case, the gas thickness is $1 \mathrm{~cm}$ and $N_{0}$ equals 20 collisions per centimetre. The result is represented with a binning of $20 \mu \mathrm{m}$ and given in percentage of $N_{0}$, on an average of 10000 tracks.

tory $*$. The $\Delta x_{i}$ of a track can then be compared to $\rho:$ a collision $i$, or a succession of collisions $i, i+1, \ldots$, having its (or their) $\Delta x_{i}, \Delta x_{i+1}, \ldots$ smaller than $\rho$ are considered by definition as lost counts. If $\nu$ is their number, the number of counted clusters per track is

$n=N-\nu$.

The mean value and its fluctuation, associated to $N_{0}$, are provided by the passage of a sufficiently large sample of tracks through the gas layer.

When diffusion is taken into account, this picture has to be changed as the clusters where $n_{i}$ is $>1$ will blow up. The initial $x_{i}$ pattern of the primary clusters is modified by quantities $k \sigma_{i}$ proportional to $\sqrt{ } x_{i}$ with $k$ drawn in a normal distribution. The new quantities $x_{i}^{\prime}=x_{i} \pm k \sigma_{i}$ are then re-ordered in an increasing $x$ sequence and their $\Delta x_{i}^{\prime}$ compared to $\rho$. In case of a secondary multiplication $n_{i}$ on the cluster $i$, this operation is repeated $n_{i}$ times at the same $x_{i}$. Eq. (1) must now be written

$n=\sum_{i=1}^{N} n_{i}-\nu$.

This picture leads us to stress the difference between a primary cluster of size $n_{i}$ and a cluster measured in presence of diffusion: the latter one consists in a sequence of electrons, all separated by less than $\rho$, which can be composed of a mixture of different primary clusters $i-2, i-1, i, i+1, \ldots$, or of part of

\footnotetext{
* The lateral diffusion may be taken into account in the same way assuming that the collection time in the detection chamber II (fig. 1) is not seriously affected by this transverse displacement.
} 
them. They will be later on referred to as "counted clusters".

It can be seen from eq. (2) that their mean n may be found greater than $N_{0}$ if the detector resolution is made sufficiently good; at the limiting case, when $\rho=$ 0 , the total number of secondary electrons is obtained:

$n=\sum_{i=1}^{N_{0}} n_{i}=N_{\mathrm{t}}$.

\subsection{Effect of the detector resolution in absence of diffusion}

In this first application, we take $\sigma_{0 \text { diff }}=0$. Therefore, we are in the situation of counting a set of primary clusters (or a set of single electrons) with a resolution $\rho$. In fig. 4 , the number of counted clusters is plotted versus the initial number of primary clusters. A variation of $N_{0}$ means either a different gas or pressure, or Lorentz factor $\gamma$ of the particle. For the sake of particle identification, as it will be analysed in the rest of the paper, let us introduce

$\Delta N=N_{\mathrm{PL}}-N_{\mathrm{MIP}}$

the expected increase in number of primary clusters for a particle on the Fermi plateau, $N_{\mathrm{PL}}$, and a minimum ionizing particle, $N_{\mathrm{MI}}$. We see from the flattening of the curves in fig. 4 that the $\Delta n$ which will be counted, corresponding to $\Delta N$, decreases as $N_{\mathrm{MIP}}$ and $\rho$ are chosen large, which is equivalent to a decrease

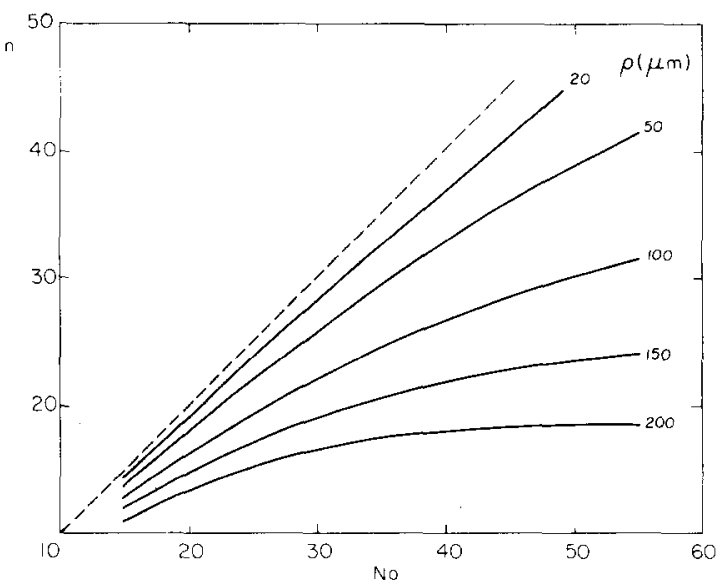

Fig. 4. $N_{0}$ is the mean number of primary collisions into $10 \mathrm{~mm}$ of gas. $n$ is the mean number of counted clusters, taking only into account the detector resolution $\rho$. This is a "zero diffusion" situation. in the measured relativistic rise. It is apparent that the gas (and its pressure) has to be chosen with a not too high $N_{\text {MIP. }}$.

\subsection{Effect of the diffusion}

Let us first consider clusters of size 1 (single electron). We have to introduce the effect of the diffusion in the situation described in fig. 4 . At a $\sigma_{0 \text { diff }}$ as large as $400 \mu \mathrm{m}$ these curves are not affected. A similar result is found in a gas thickness of $20 \mathrm{~mm}$. This shows that diffusion will not introduce any further loss or gain in a single electron counting than the one already produced by the detector resolution.

This situation changes as soon as the cluster size differs from 1, as it is shown in the following way: we randomly draw, among a given number $N_{0}$ of primary clusters a known proportion of them to which a size 2 is assigned. Fig. 5 shows for a value of $\sigma_{0 \text { diff }}$ of

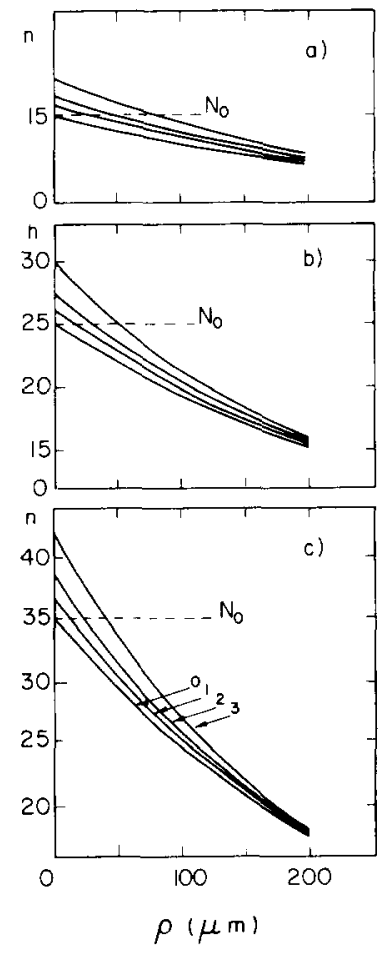

Fig. 5. The number of counted clusters $n$, in $l=10 \mathrm{~mm}$, versus the detector resolution $\rho$, in presence of a diffusion $\sigma_{0 \text { diff }}=200 \mu \mathrm{m}$. The different sets of curves $(\mathrm{a}, \mathrm{b}, \mathrm{c})$ correspond to $N_{0}=15,25,35$ primary collisions (dashed horizontal line). The effect of a given cluster size distribution is shown on the different curves $0,1,2,3$ where populations of cluster size 1 and 2 are respectively fixed to $100 \%, 95 / 5 \%$, $90 / 10 \%, 80 / 20 \%$. 


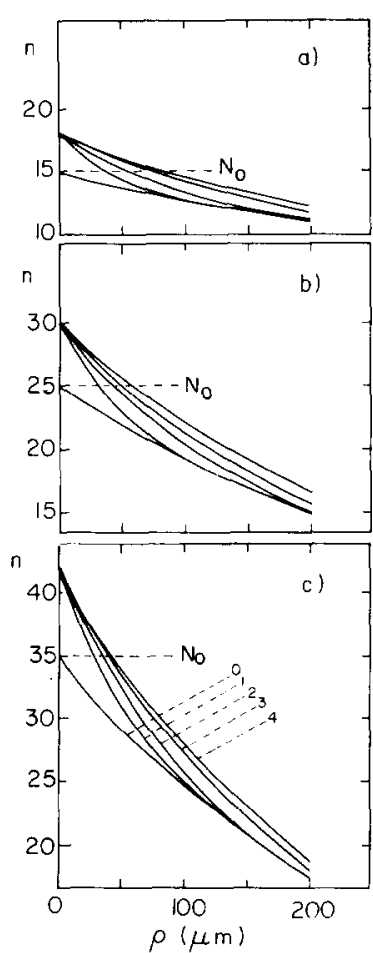

Fig. 6. Effect of different diffusion constant on the member of counted clusters $n$, versus the detector resolution $\rho$. The cluster size distribution is fixed to $80 / 20 \%$ of sizes 1 and 2 . $(\mathrm{a}, \mathrm{b}, \mathrm{c})$ correspond to $N_{0}=15,25,35$ primary collisions in $l=10 \mathrm{~mm}$ of gas (horizontal dashed lines). The total number of electrons are respectively $18,30,42$. The curves $0,1,2,3$, 4 correspond to $\sigma_{0 \text { diff }}=0,50,100,200,400 \mu \mathrm{m}$.

$200 \mu \mathrm{m}$, that the diffusion blows up these clusters of size 2 in such a way that the number of counted clusters may now exceed $N_{0}$ for a resolution $\rho \lessgtr$ $100 \mu \mathrm{m}$. The curves tend to overlap at large $\rho$ the curve $100 \%$ cluster of size $=1$, equivalent to $\sigma_{0 \text { diff }}=$ 0 . The effect of $\sigma_{0 \text { diff }}$ is also shown for a fixed initial CSD on fig. 6.

\section{Energy loss and cluster size distribution in argon}

We will now describe the part of the simulation devoted to the energy loss in a gas commonly used in these detectors, i.e. argon. The program organization together with the details of the accounted atomic mechanisms are grouped in the appendices B-G.

We are faced with the problem of getting the variation of the number of primary clusters as a function of $\gamma$ together with the associated cluster size distributions, given in number of electrons. This implies a simulation program different from those developped up to now [8], [9], where the total loss of a particle through a gas layer of thickness $l$ was provided in energy unit by a summation of the $E_{i}$.

Let us remark that the only experimental comparison for a simulation in pure argon may be found, within limits discussed in sect. 6, from $\mathrm{d} E / \mathrm{d} x$ measurements performed in a mixture of argon and few percent of methane. This is precisely the gas mixture used in the EPI detector, to which we will compare our $\mathrm{d} E / \mathrm{d} x$ predictions.

\subsection{Simulation in terms of number of Electrons Locally Deposited-ELD model}

Such an approach is obviously requested by the nature of the cluster size distribution. The mechanism of the loss is therefore considered from a local point of view, each energy transfer $E_{i}$ being converted in a certain number of secondary electrons. In this model, the $E_{i}$ computation is based on the work of Chechin et al. [10] (appendix C). The experimental photoabsorption cross section [11] is used in the calculation of the oscillator strengths and in the calculation of the complex refractive index (appendix D). The oscillator strengths allow to take into account the atomic binding effects accordingly to the different shells, K, L and M. The only transfers $E_{i}$ of interest are those greater than $I_{0}$, the first ionization potential of argon (15.75 eV) (see sect. 6). In the case of a minimum ionizing particle, table 1 shows that $71 \%$ of the transfers have their energies between 15.7 and $30 \mathrm{eV}, 85 \%$ between 30 and $45 \mathrm{eV}$; the fifteen per cent, larger than $45 \mathrm{eV}$ are spread over a very large energy range.

Let us consider first the $71 \%$ of the transfers $E_{i}$ with energies between 15.75 and $30 \mathrm{eV}$. They released a single electron from the $M$ shell which is left with a kinetic energy smaller than $I_{0}(15.75 \mathrm{eV})$ and cannot multiply anymore in pure argon. The remaining $29 \%$ of the transfers $(>30 \mathrm{eV})$ released electrons, of kinetic energy $K_{i}$ allowing a further multiplication. Among these transfers, we have taken into account (as a zero order step), of the different Auger [12] effects occurring on $K$ and $L$ shells in argon when $E_{i} \geqslant 250 \mathrm{eV}$ (appendix E). In these cases, several electrons are produced from one transfer.

The electrons, with an initial kinetic energy $K_{i}$ are going to loose their energy by successive interactions with the gas atoms according to ionizing and excitating impacts. The secondary multiplication is only 
Table 1

Referred to the total number of primary collision, percentage of those having their energy transfers included in steps of $15 \mathrm{eV}$ (pure argon). The first set of energies implies transfers on the $\mathrm{M}$ shell; the second set, on the $\mathrm{L}$ shell. Values calculated for a minimum ionizing $(\gamma=4)$ and "on the Fermi plateau" $(\gamma=1000)$ particles with, respectively $N_{0}=26.6$ and 34.96 .

\begin{tabular}{|c|c|c|c|c|c|}
\hline $\begin{array}{l}\text { Energy } \\
\text { transfer }(\mathrm{eV}) \\
\text { between }\end{array}$ & $\begin{array}{l}\gamma=4 \\
(\%)\end{array}$ & $\begin{array}{l}\gamma=1000 \\
(\%)\end{array}$ & $\begin{array}{l}\text { Energy } \\
\text { transfer (eV) } \\
\text { between }\end{array}$ & $\begin{array}{l}\gamma=4 \\
(\%)\end{array}$ & $\begin{array}{l}\gamma=1000 \\
(\%)\end{array}$ \\
\hline $15-30$ & 70.9 & 69.3 & $225-240$ & 0.1 & 0.1 \\
\hline $30-45$ & 14.1 & 13.7 & $240-255$ & 0.4 & 0.5 \\
\hline $45-60$ & 1.9 & 1.7 & $255-270$ & 0.6 & 0.8 \\
\hline $60-75$ & 1.6 & 1.5 & $270-285$ & 0.6 & 0.7 \\
\hline $75-90$ & 1.1 & 1.2 & $285-300$ & 0.5 & 0.6 \\
\hline $90-105$ & 0.9 & 0.9 & $300-315$ & 0.4 & 0.5 \\
\hline $105-120$ & 0.7 & 0.8 & $315-330$ & 0.3 & 0.4 \\
\hline $120-135$ & 0.5 & 0.6 & $330-345$ & 0.3 & 0.4 \\
\hline \multirow[t]{3}{*}{$135-150$} & 0.4 & 0.5 & $345-360$ & 0.3 & 0.3 \\
\hline & & & $360-375$ & 0.2 & 0.3 \\
\hline & & & $>375$ & 3.2 & 4.3 \\
\hline
\end{tabular}

effective in ionizing impacts and will be stopped when $K_{i}$ will become smaller than $I_{0}$. The excitation and ionization cross sections are variable over the energy range above $I_{0}$, especially in its lower part where excitation lines are observed (appendix F). Usually, the number $n_{i}$ of secondaries is calculated as $K_{i} / \bar{w}_{0}$, where $\bar{w}_{0}$ is the mean work to create an electron-ion pair. Its value, $26.4 \mathrm{eV}$ [13], larger than $I_{0}$, takes already into account the inefficient losses by excitation impacts.

Division by $\bar{w}_{0}$ has been used in the ELD model for $K_{i} \geqslant 1000 \mathrm{eV}$. For $K_{i}$ between 15.7 and $1000 \mathrm{eV}$, that is about $28 \%$ of the transfers, $\bar{w}_{0}$ has to be corrected: firstly, the excitation cross section increases when $K_{i}$ becomes smaller than $\sim 50 \mathrm{eV}$ [14]; secondly, after the few expected shocks (1 to $5)$, the energy remainders, depending on whether they are smaller or greater than $I_{0}$, may yield an uncertainty of one electron, which represents an important relative error. A model is proposed in appendix $F$ for the range $15.7-1000 \mathrm{eV}$, which leads to a $\bar{w}$ larger than $26.4 \mathrm{eV}$ at small values of $K_{i}$, but finds back the usual value of $\bar{w}_{0}$ at $K_{i} \geqslant 1000 \mathrm{eV}$ (see fig. 25 in appendix $F$ ).

An other parameter is the Fano factor, related to the fluctuation of $n_{i}$ in a particular gas. Its expression is [15] $\left(\delta n_{i}\right)^{2}=F n_{i}$. In argon, $F$ is equal to 0.18 and is not very sensitive to $K_{i}$. Comparable results, $F=$ 0.18 , were observed by means of energy transfers greater than $260 \mathrm{eV}$ (photons) into a proportional counter [16] and of $\alpha$-particle losses in an ionization chamber [17], where the local transfers are smaller.
The results of the simulation agree with these features (fig. 24).

\subsection{Results}

\subsubsection{Cluster size distribution}

Table 2 indicates the percentages of the different sizes for particles at the minimum and on the Fermi plateau ( $\gamma=4$ and 1000). One finds that $80.2 \%$ of the clusters have a size 1 and $7.7 \%$ a size 2 . The slight bump observed at sizes $8-12$ is due to the rise of photoabsorption cross sections on the $\mathrm{L}$ shell (fig. 22. Then, a small probability exists for finding a cluster size up to 100 electrons $\left(\sim 2 \times 10^{-5}\right.$ in this case). The maximum transfer introduced as a cut in the program was fixed at $15 \mathrm{keV}$; the range of such an electron is sufficient that it may escape from a $1 \mathrm{~cm}$ thick detection layer.

In order to estimate the influence of the relative population of sizes 1 and 2 on the counting of clusters, secondary multiplication has been differently calculated: the $n_{i}$ are taken as the integer part $\left[K_{i} / \bar{w}_{0}\right]$. The sizes 1 and 2 are found now in the percentages of 84.3 and $3.4 \%$. This enrichment of size 1 , together with a slight modification for sizes $>8$, will be of very little influence on the number of counted clusters. This simpler method might be used to calculate the CSD.

\subsubsection{Deposited energy}

In the ELD simulation, the "energy" lost through a gas layer of thickness $l$ is obtained by the summa. 
Table 2

Cluster size distribution for a minimum ionizing and "on the Fermi plateau" particles crossing $10 \mathrm{~mm}$ of argon. The populations are given in percentage of the total number of primary collisions, which are on the average 26.6 and 34.96 at $\gamma=4$ and 1000 . The secondary multiplication is calculated in (I), using a "Fano" model; in (II) "in teger part".

\begin{tabular}{|c|c|c|c|c|}
\hline \multirow{2}{*}{$\begin{array}{l}\text { Cluster size } \\
\text { (number of } \\
\text { electrons) }\end{array}$} & \multicolumn{2}{|c|}{ (I) } & \multicolumn{2}{|c|}{ (II) } \\
\hline & $\begin{array}{l}\gamma=4 \\
(\%)\end{array}$ & $\begin{array}{l}\gamma=1000 \\
(\%)\end{array}$ & $\begin{array}{l}\gamma=4 \\
(\%)\end{array}$ & $\begin{array}{l}\gamma=1000 \\
(\%)\end{array}$ \\
\hline 1 & 80.2 & 78.2 & 84.1 & 82.2 \\
\hline 2 & 7.7 & 7.4 & 3.4 & 3.1 \\
\hline 3 & 2.0 & 2.1 & 2.1 & 2.2 \\
\hline 4 & 1.3 & 1.4 & 1.3 & 1.4 \\
\hline 5 & 0.8 & 0.8 & 0.8 & 0.9 \\
\hline 6 & 0.6 & 0.7 & 0.6 & 0.7 \\
\hline 7 & 0.5 & 0.6 & 0.4 & 0.4 \\
\hline 8 & 0.6 & 0.8 & 0.3 & 0.3 \\
\hline 9 & 0.8 & 1.1 & 0.8 & 1.1 \\
\hline 10 & 0.9 & 1.2 & 1.3 & 1.7 \\
\hline 11 & 0.7 & 0.9 & 0.8 & 0.9 \\
\hline 12 & 0.5 & 0.7 & 0.5 & 0.5 \\
\hline 13 & 0.4 & 0.5 & 0.4 & 0.5 \\
\hline 14 & 0.3 & 0.4 & 0.3 & 0.4 \\
\hline$\Sigma 2-7$ & 12.9 & 13.0 & 8.6 & 8.7 \\
\hline$\Sigma 8-20$ & 5.3 & 6.9 & 5.6 & 7.1 \\
\hline$\Sigma>20$ & 1.4 & 1.65 & 1.50 & 1.75 \\
\hline overflow & 0.2 & 0.25 & 0.20 & 0.25 \\
\hline
\end{tabular}

tion of numbers of electrons, $n_{i}$, created along this path, each of these $n_{i}$ having been calculated at a local transfer. Let us remark that the pulse height measured from a multiwire chamber is anyhow proportional to a number of charges released near the wire, after multiplication of these $n_{i}$ initial electrons.

Therefore in order to get the deposited energy, the only step to be added to the simulation is the fluctuation of the number of charges inherent to the avalanche process [18]. The standard deviation associated to this fluctuation is discussed in appendix G. Is it now possible to get this loss in energy unit? The relation between the number of initial electrons and the associated pulse height is usually obtained by a calibration with a photon source releasing very locally a known energy, for example $5.9 \mathrm{keV}$ with an ${ }^{55} \mathrm{Fe}$ source. In this case, as division of this energy by $\bar{w}$ is meaningful, so it is for the number of electrons, calculated so far, (223). This proportionality can be kept if care is taken to avoid saturation effect in the working range of charges created around the wire. For an ionizing particle, if such a calibration is still valid in terms of number of electrons, its scaling in energy unit becomes approximate as the loss is composed in this case of a succession of small transfers where the division by $\bar{w}$ has been found inadequate.

We are now in a position to compare our calculations with a pulse height measurement. In fact, this can be a first estimation of the level of confidence to the ELD predictions about primary mechanisms, that is, size and number of clusters per unit length as a function of $\gamma$.

Fig. 7 shows, in (a) a distribution of the total number of electrons deposited in $10 \mathrm{~mm}$ of argon $(\gamma=$ 1000 ) and in (b) the effect of an avalanche fluctuation defined by the relation (16) (appendix $\mathrm{G}$ ), where $\left(\sigma_{A} / A\right)^{2}=0.66$ (Polya distribution with $\left.\theta=1 / 2\right)$. The mean and most probable number of electrons, $N_{\mathrm{t}}$ and $N_{t}^{\mathrm{pp}}$, are found equal to 70 and 42 , at $\gamma=4$. The resolution, (fwhm) is then $100 \%$. The most probable value can be compared to a Lehraus measurement [2] in a mixture of argon and 5\% methane which leads to $N_{\mathrm{t}}^{\mathrm{PP}} \cong 48$.

\subsubsection{Relativistic rise}

We present in fig. 8 the relativistic rise $I / I_{0}$ predicted for the mean and the most probable values of the total number of electrons. The rise is referred to the minimum of ionization. In order to be compared to the EPI results [2], the most probable values were calculated as a truncated mean, calculated as the 50\% smallest of a sample of 100 cells. The experimental points are indicated on the figure. We must remark that the determination of these most probable values depends on the choice of the truncation, (curves $\mathrm{C} 1$

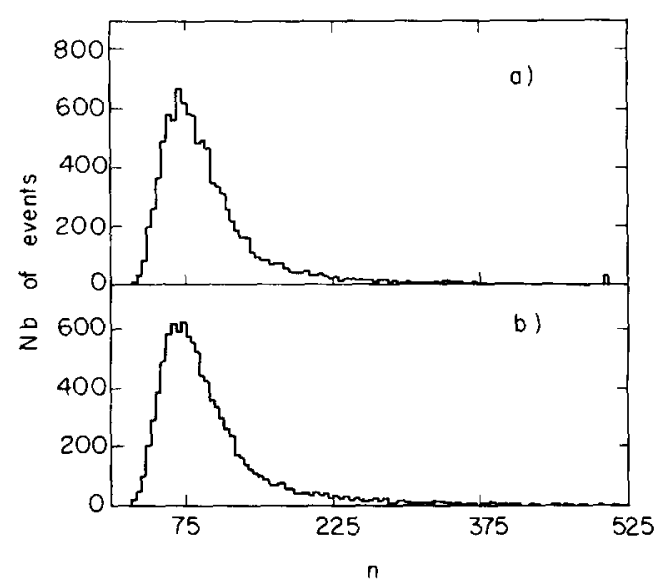

Fig. 7. (a) Distribution of the total number of electrons deposited per track in $10 \mathrm{~mm}$ of argon $(\gamma=1000)$ (according to the model described in the appendices). (b) Distribution obtained after superposing an avalanche fluctuation. 


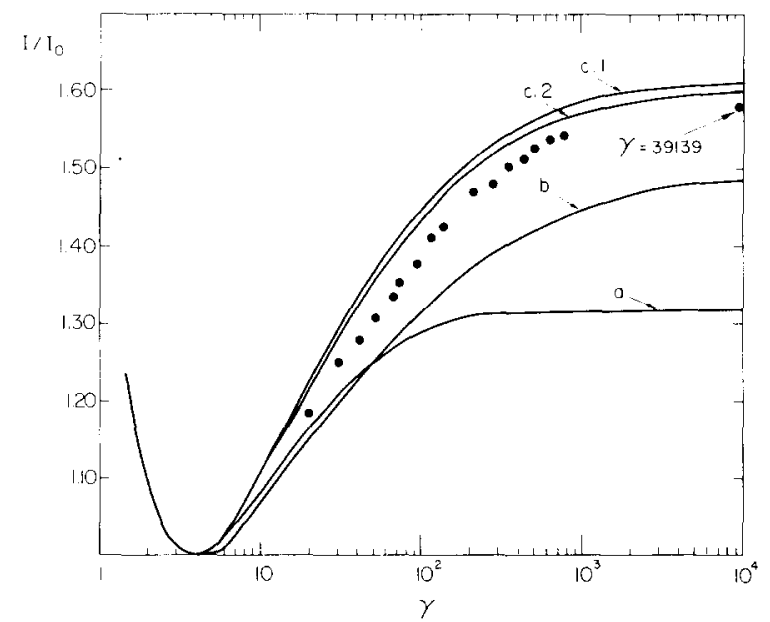

Fig. 8. Relativistic rise of the ionization in a $10 \mathrm{~mm}$ layer of argon: (a) Number of primary clusters. (b) Mean number of deposited electrons (an avalanche fluctuation is included). (c) Most probable number of deposited electrons. $40 \%$ smallest values: $\mathrm{C} 1 ; 50 \%$ smallest values: $\mathrm{C} 2$. Circles: most probable $\mathrm{d} E / \mathrm{d} x$ measurements from the EPI (argon plus $5 \%$ methane) [2].

and $\mathrm{C} 2$ in fig. 8) and, for the experimenter, of the cut which is introduced by the overflows inherent to the usual measuring device as an analog-to-digital converter. From this point of view, the reported fit may be esimated as satisfactory; it would be of little interest to look for further improvements with respect to other experimental working conditions (methane) not considered so far into the simulation.

On the same graph, the relativistic rise of the number of primary clusters is also drawn. The striking facts are: (1) this curve saturates more rapidly, at a $\gamma$ of $\sim 200$, than those relative to deposited energy and (2) the maximum ratio reached on the Fermi plateau is 1.32 , while it rises to 1.48 and 1.60 respectively, for the mean and most probable energy losses.

Let us briefly comment on these two points.

(1) It is known [10b], that the number of collisions per unit length at a given transferred energy increases at first with $\gamma$, and then saturates because of the density effect (fig. 21). This saturation begins at small transfers, interesting the $M$ shell, to reach at higher $\gamma$ the $\mathrm{L}$ and finally the $\mathrm{K}$ shells. A saturation of the number of transfers is then observed at first, while the transferred energy continues to increase. The prediction gives an $11 \%$ increase for the mean energy transfer when $\gamma$ varies from 4 to 1000 . Fig. 9 shows the variation versus $\gamma$ of the percentage $p_{M}$ of transfers included between 15.7 and $50 \mathrm{eV}$ (M shell)

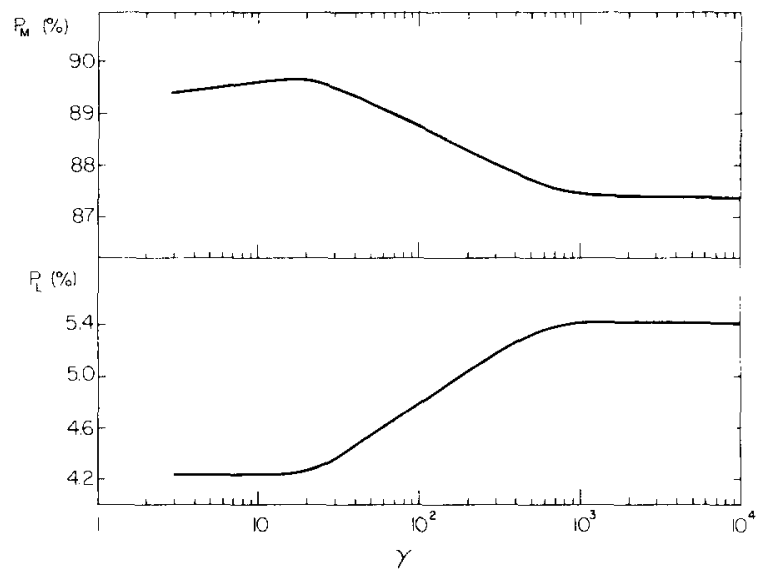

Fig. 9. Relative relativistic rise of the number of transfers: $p_{\mathrm{M}}$ : percentage of transfers on the $\mathrm{M}$ shell $(15.7-50 \mathrm{eV}), p_{\mathrm{L}}$ : percentage of transfers on the L shell $(250-400 \mathrm{eV})$.

and $p_{\mathrm{L}}$, the percentage of those between 250 and $400 \mathrm{eV}$ ( $\mathrm{L}$ shell), with respect to the total number of transfers. The saturation effect of the $M$ shell is apparent in the relative decrease of $p_{M}$ from $\gamma=20$, while $p_{\mathrm{L}}$ begins to increase from $\gamma \simeq 20$ and saturates at $\gamma \simeq 1000$.

(2) On the other hand, it can be calculated from the percentages listed in table $2\left(\gamma=4, N_{0}=26.61\right)$ that one has to consider the clusters of size 1 to size 12 to yield a total number of electrons of 42 , which is the most probable value expected in $1 \mathrm{~cm}$ of argon. Among these 42 electrons, 21.3 originates from a cluster of size 1, 10 from clusters of sizes 2-7 and 10.7 from clusters of sizes $8-12$ (L shell). In other words, if one speaks in terms of primary collisions, a most probable event will consist on the average of respectively $21,3-4$ and 1 collisions, which initiate clusters of sizes $1,2-7$ and $8-13$ electrons (see also fig. 21).

It can be suggested that the continuous increase of $p_{\mathrm{L}}$ is acting more effectively on the relativistic rise of the most probable energy than on the mean energy. In this latter case, the occurrence of larger transfers $(>500 \mathrm{eV})$, where the density effect is not yet efficient, has too much weight and masks these smaller variations.

\section{Cluster counting in a one centimeter layer of argon}

We have now all the elements to simulate a cluster counting experiment. We shall first discuss the results relative to a $1 \mathrm{~cm}$ layer of argon; the diffusion is fixed 


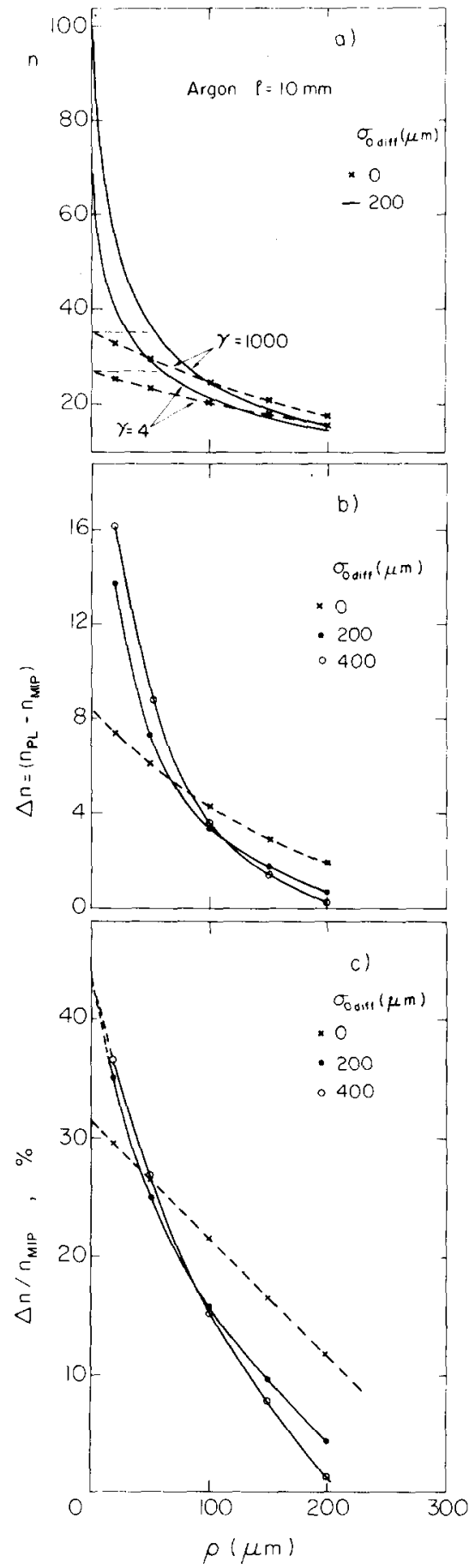

Fig. 10. (a) Counted clusters $n$ in $10 \mathrm{~mm}$ of argon versus the detector resolution for $\gamma=4$ and 1000 . The horizontal dashed lines indicate the number of primary clusters in these both cases. (b) $\Delta n$ : difference between the number of counted clusters on the plateau and at the minimum. At $\rho=$ 0 , this difference equals 30.81 electrons. (c) Relative variation of this difference $\Delta n$ with respect to the expected number at the minimum of ionization. On these graphs, the at $\sigma_{0 \text { diff }}=200 \mu \mathrm{m}$. A thickness of $2 \mathrm{~cm}$ does not show any significant differences. On every plot, a primary cluster counting is represented as a "reference curve" (drawn with crosses $X$ ). It implies $\sigma_{0 \text { diff }}=0$, and therefore, only the detector resolution is taken into account.

\subsection{Number of counted clusters as a function of the detector resolution}

In fig. 10, the number of counted clusters is plotted versus the detector resolution in the two extreme cases, a particle on the Fermi plateau and at the Minimum of Ionization (MIP). The curves have been extrapolated at $\rho=0$ to their respective total number of electrons $N_{\mathrm{t}}$. From these curves, one can draw the variation of $\Delta n=n_{\mathrm{PL}}-n_{\mathrm{MIP}}$ and $\Delta n / n_{\mathrm{MIP}}$, respectively the absolute and relative increases of the number of electrons due to the relativistic rise.

The effect of the diffusion on the CSD allows to count a number of clusters larger than the one primarily deposited, up to a resolution of 50-60 $\mu \mathrm{m}$. However, n drops rapidly from its "Landau" value $N_{\mathrm{t}}$ as soon as a resolution as good as $20 \mu \mathrm{m}$ is applied. The values of $n, \Delta n$, and $\Delta n / n_{\mathrm{MIP}}$ are at first higher, then lower than the reference curves, as much as the diffusion coefficient is large. This effect is clearly related to the spread of cluster sizes inherent to the argon CSD. A good resolution allows to count an important fraction of the secondary electrons, but due also to this larger number of electrons, the probability to bridge them in longer clusters is increased when the resolution degrades. In fact, this crossing effect was not observed in fig. 5 , where the cluster size abundance were arbitrarily fixed at $85 \%$ for size 1 - i.e. about the same value as in argon, but the $15 \%$ remaining were of size 2 .

In order to estimate the influence of the large cluster sizes $(>12)$ a selection of events has been done by counting the clusters only when the total number of electrons, $N_{\mathrm{t}}$, was smaller than a fixed value. When this cut is taken as the most probable value (curves 1 in fig. 11), the counting becomes much more similar to a primary counting, at the price of a probability of such an event now smaller than one.

curves $(+)$ describe a "pure" cluster counting experiment without any blowing effect of the real clusters under the effect of diffusion ( $\sigma_{0}$ diff $=0$ ). 


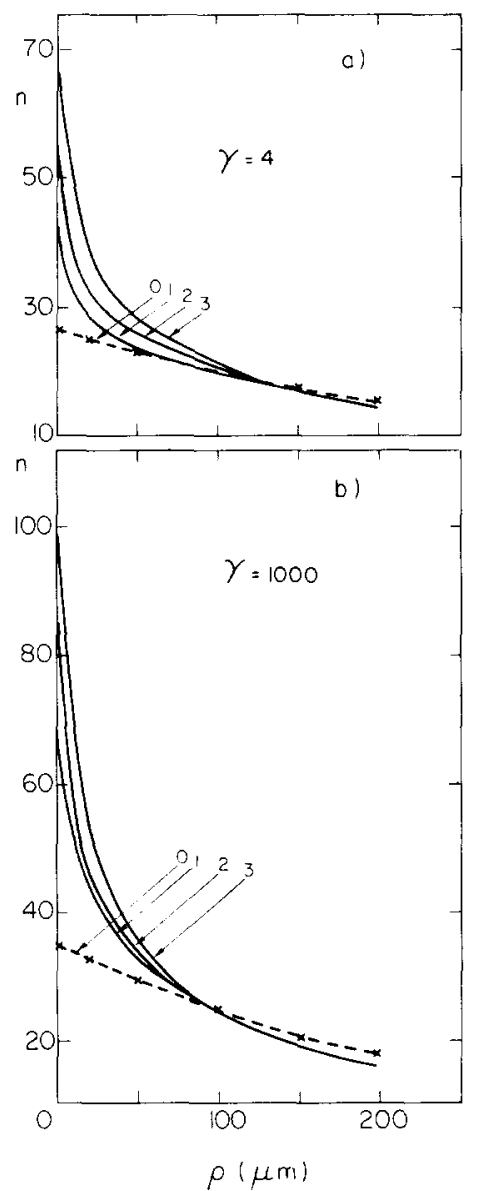

Fig. 11. Counted cluster in $10 \mathrm{~mm}$ of argon on selected events: Curve 0 : primary clusters $\left(\sigma_{0 \text { diff }}=0\right)$. Curve 1: events with their total number of electrons smaller than the most probable one. Their probability is then $\sim 0.3$. Curve 2 : events belonging to the lower half of the $N_{\mathrm{t}}$ spectrum. Their probability is 0.5 . Curve 3 : all the events.

\subsection{Counted clusters distribution and standard devia- tion}

As already pointed out, the number of primary clusters fluctuates according to a Poisson law determinted by its mean $N_{0}$. The standard deviation is then fixed to $\sqrt{ } N_{0}$. In fig. 12 , we present the computed counted clusters distribution at $\sigma_{0 \text { diff }}=0$. At $\rho=0$ the Poisson curve is found but as soon as finite resolutions $\rho$ are applied, the distributions fit to gaussian distributions defined by their means $n(\rho)$ and a standard deviation $\sigma_{1}(\rho)$, now different from $\sqrt{ } n(\rho)$. We have plotted for comparison the Poisson distributions defined by the same $n(\rho)$. In fig. 13, distributions are drawn for $\sigma_{0 \text { diff }}=200 \mu \mathrm{m}$. Now at $\rho=0$, the Landau curve is found. Then, as soon as $\rho$ differs from 0 , the Landau tail is nearly eliminated. The distribution $\rho=20 \mu \mathrm{m}$ still shows a small kurtosis, remainder of the very assymmetric Landau spectrum, and is also wider than the Poisson distribution $n(20)$. Then, as $\rho$ increases, the distributions become gaussian as previously, but for $\rho=50 \mu \mathrm{m}$, the fit with the poissonian $n(50)$ is excellent. $\sigma_{1}(\rho)$ is plotted versus $\rho$ in fig. 14 together with the corresponding Poissonian standard deviation $\sqrt{ } n(\rho)$. At $\sigma_{0 \text { diff }}=0, \sigma_{1}(\rho)$ is always smaller than $\sqrt{ } n(\rho)$, the crossing point being at $\rho=0$, where $\sigma_{1}(0)=\sqrt{ } N_{0}$ by definition. For $\sigma_{0 \text { diff }}=200 \mu \mathrm{m}, \sigma_{1}(\rho)$, at first greater than $\sqrt{ } n$, tends towards the reference $\sigma_{1}(\rho)$. Its crossing point with $\sqrt{ } n(\rho)$, where the distribution is Poissonian, is found as already mentioned at $\rho \simeq 50 \mu \mathrm{m}$, but for $n(\rho)$ precisely equals to $N_{0}$. This very particular coincidence has been checked for different $N_{0}$ and $\sigma_{0 \text { diff }}$. The crossing points are then found at different values of $\rho$. It seems peculiar to find back the characteristic Poissonian behaviour of the primary process in a counting where a diffusion fluctuation and a cut have distorted this initial distribution, precisely when the number of counts coincides with those of the primary process. This feature will be also considered in the detector size optimization.

Finally, in fig. 15, we present the relative precision $\sigma_{1}(\rho) / n(\rho)$. As $\rho$ increases, the precision begins to improve, $\left(\sigma_{1} / n\right.$ decreases) to reach an optimum for $\rho \approx$ $100 \mu \mathrm{m}$. From the preceding remarks, an horizontal line drawn in this graph at $\sigma_{1} / n=1 / \sqrt{ } N_{0}$ has to intercept these curves at points where $n(\rho)=N_{0}$. It will be shown later on, that the optimum size detector to achieve a given particle separation is not found for the $\rho$ corresponding to the optimum of the precision, but precisely for a $\rho$ satisfying to $n(\rho)=N_{0}$ and its Poissonian distribution. At the optimum $\rho(\simeq 100-$ $150 \mu \mathrm{m}), \Delta \mathrm{n}$ has in fact dropped to much with respect to the gain in precision (fig. 10b, c).

Let us however remark that for experiments where only the absolute value of the loss is of interest (charge multipicity for example), an optimum precision can be reached with $\rho \simeq 150 \mu \mathrm{m}$ and $\sigma_{0 \text { diff }}=$ $400 \mu \mathrm{m}$ : in these conditions a fwhm of $\sim 40 \%$ is achieved in a $10 \mathrm{~mm}$ layer of argon instead of the $100 \%$ given by pulse height measurement.

\subsection{Detector length}

In fig. 16 is shown the variation with $\gamma$ of the mean number of counted clusters in a layer of $10 \mathrm{~mm}$ of argon, at different detector resolutions. As $\rho$ 

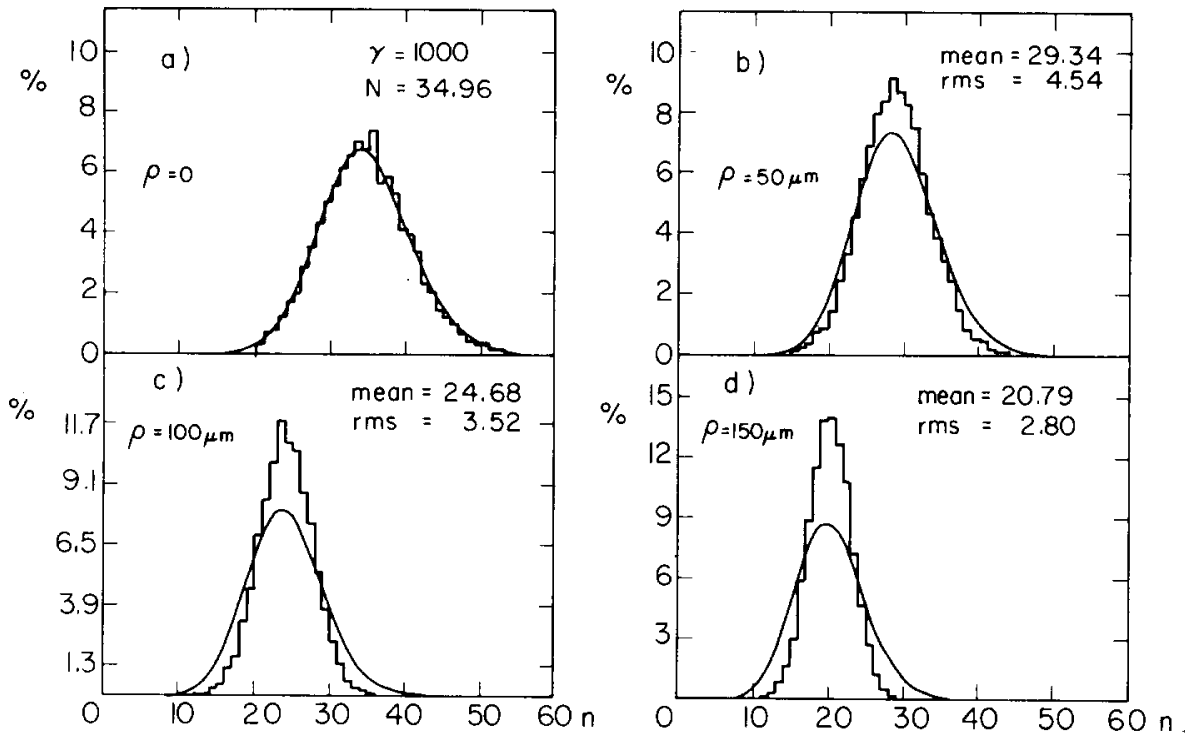

Fig. 12. Shapes of the distribution of counted clusters for different detector resolutions $\rho$. If $n(\rho)$ is the mean value found for a resolution $\rho$, the Poisson distribution having the same mean is superimposed by a continuous line, $\sigma_{0}$ diff $=0(L=10 \mathrm{~mm}$ in argon).
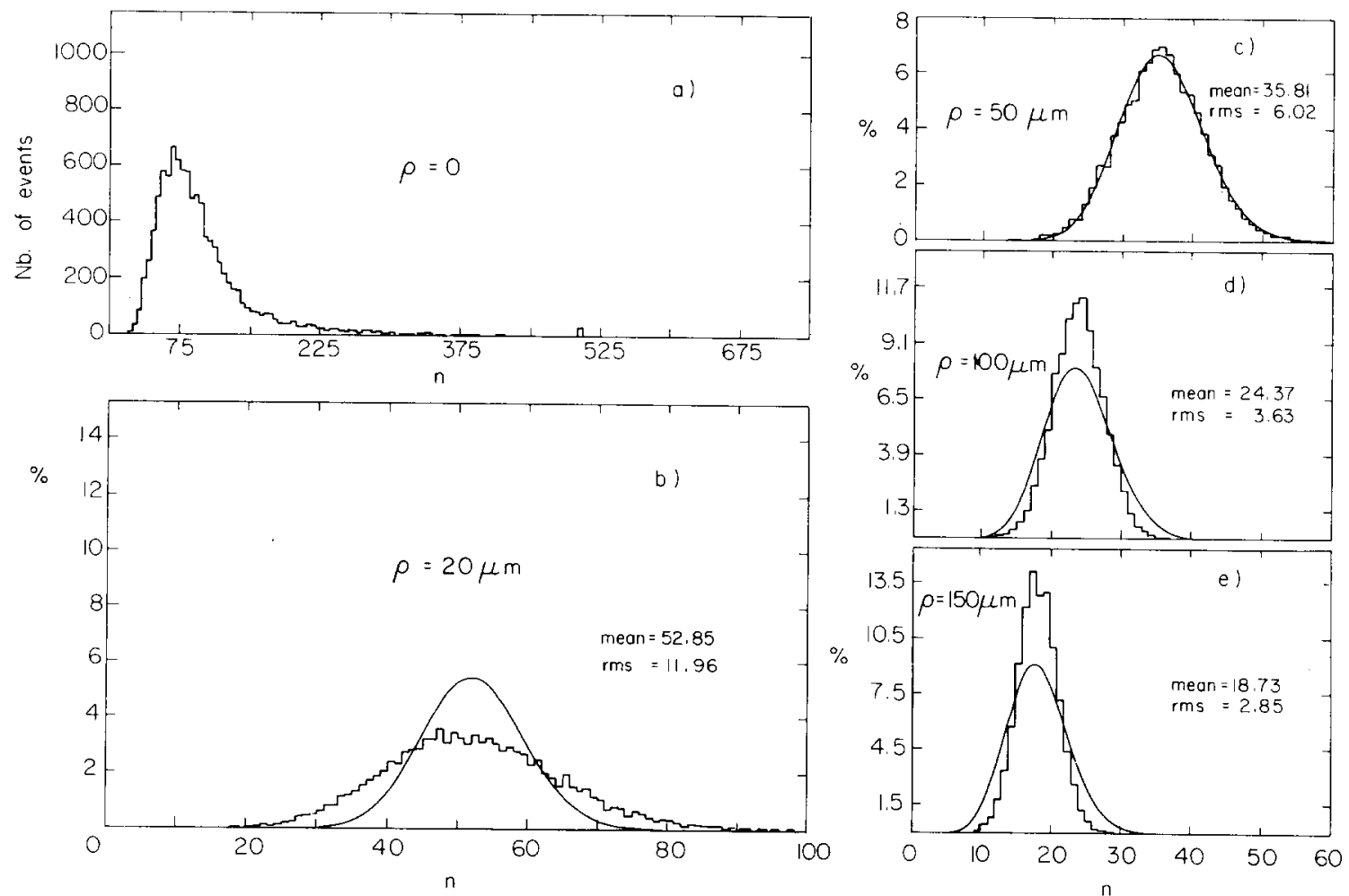

Fig. 13. Same as fig. 13, but $\sigma_{0 \text { diff }}=200 \mu \mathrm{m}$. 


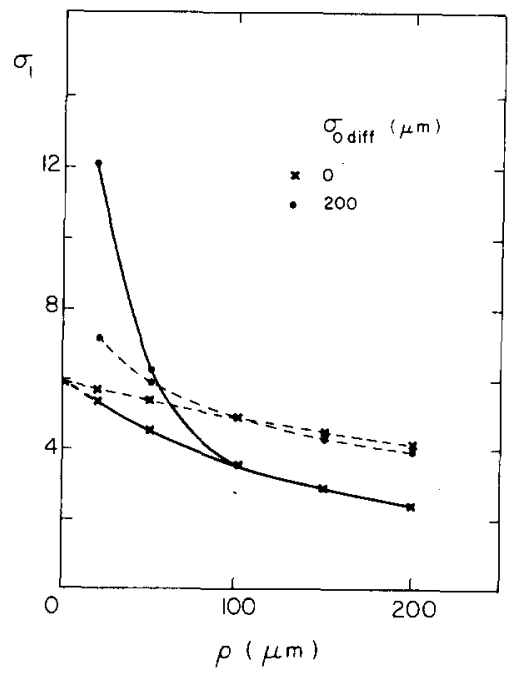

Fig. 14. Standard deviation of the distribution of counted clusters versus the detector resolution $(10 \mathrm{~mm}$ in argon, $\gamma=1000, N_{0}=35$ electrons). Full line: computed values corresponding to gaussian distribution of means $n(\rho)$. Dotted line: $\sqrt{ } n(\rho)$ that is, the standard deviation of a Poisson-like counting.

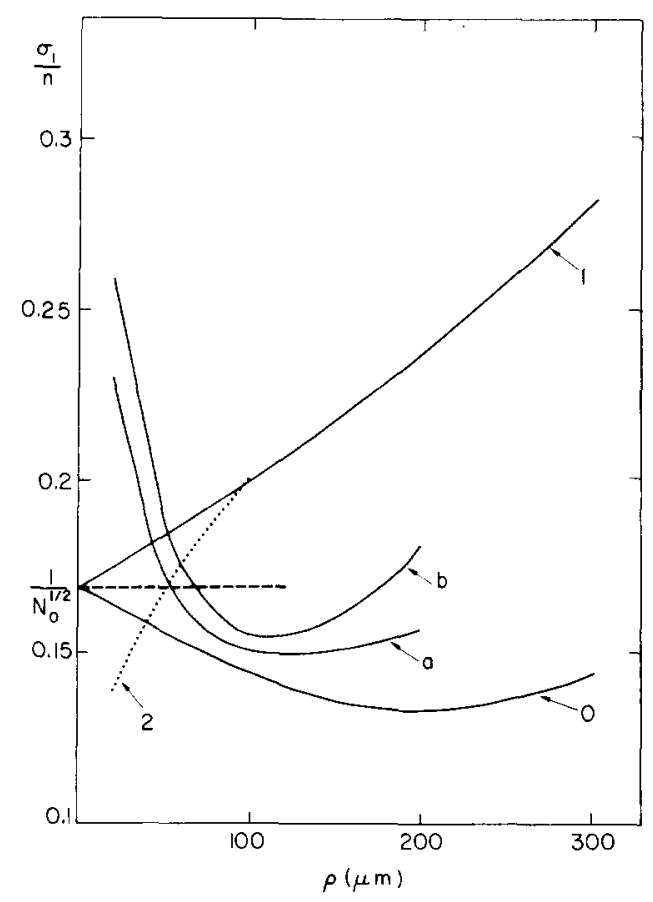

Fig. 15. Relative precision of a cluster counting in $10 \mathrm{~mm}$ of argon $\left(\gamma=1000, N_{0}=35\right)$. Curve $0: \sigma_{0 \text { diff }}=0$; Curves $\mathrm{a}, \mathrm{b}$ : $\sigma_{0 \text { diff }}=200$ and $400[\mu \mathrm{m}]$; Curve $1:$ represents the variation of $1 / \sqrt{ } n(\rho)$ at $\sigma_{0 \text { diff }}=0$; Curve 2: idem, but at $\sigma_{0 \text { diff }}=$ $200 \mu \mathrm{m}$. The intercept of curves a and 2 is found at $n(\rho)=$ $N_{0}$.

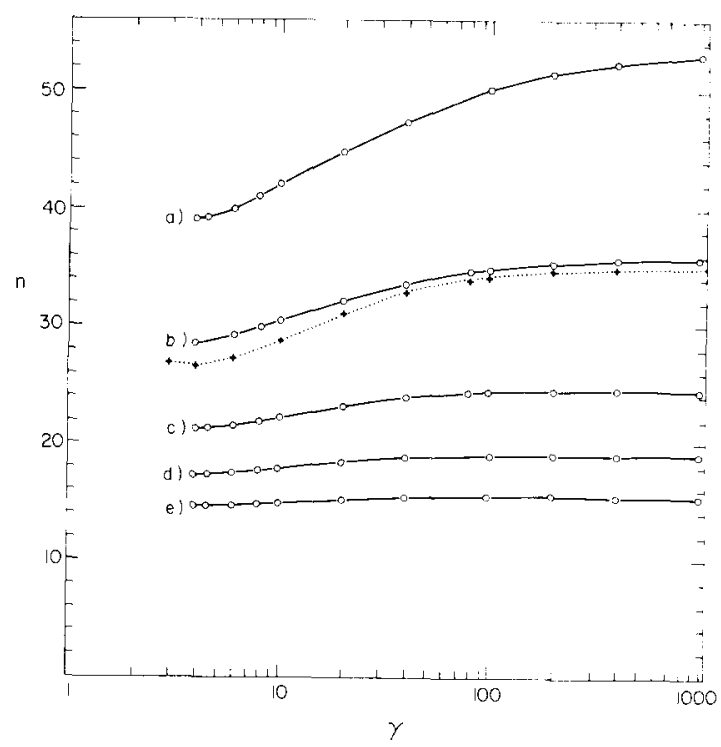

Fig. 16. Variation of the counted clusters in $10 \mathrm{~mm}$ of argon along the relativistic rise. Curves $a, b, c, d$, e correspond to the detector resolutions $20,50,100,150,200 \mu \mathrm{m}$. The diffusion is $\sigma_{0 \text { diff }}=200 \mu \mathrm{m}$. The dashed curve $(+)$ is a primary cluster counting, $\sigma_{0 \text { diff }}=0$ and $\rho=0$.

increases, the Fermi plateau is reached at smaller $\gamma$.

We have estimated the number $m$ of $10 \mathrm{~mm}$ cells necessary to achieve a proton-kaon separation using the cluster counting technique. If $n_{\mathrm{p}}(\rho)$ and $n_{\mathrm{K}}(\rho)$ are the values predicted at a given momentum $p, m$ is determined as

$n_{\mathrm{K}}-n_{\mathrm{p}}=\alpha \sigma_{\mathrm{m}}$,

where

$\sigma_{m}=\sigma_{1}(\rho) / \sqrt{ } m$,

as the $\sigma_{1}$ distributions are Gaussian-like. $\alpha$ is the number of $\sigma_{m}$ characterizing the confidence level wanted in the separation. The results (fig. 18) are calculated with $\alpha=2$. More powerful statistical techniques such as a maximum likelihood method might be used but it requires the previous knowledge of expected spectra, difficult to obtain in a real size detector over the full $\gamma$ range. For comparison, we present also the $m$ variation predicted from a $\mathrm{d} E / \mathrm{d} x$ measurement. Then, we take as reference not a $10 \mathrm{~mm}$ layer (Landau distribution) but a distribution obtained as the 50\% smallest measurements among 100 traversed cells. Such a distribution is now Gaussian-like, $\Sigma_{100}$ being its standard deviation. Its mean may be considered as the most probable energy lost in $100 \mathrm{~cm}$ of argon and will be independent of variations of $m(m>100)$. We 
used the relation

$N_{\mathrm{K}}-N_{\mathrm{p}}=\alpha \frac{\Sigma_{100}}{\sqrt{ } m}$,

where $m$ is now the number of cells of length corresponding to $\Sigma_{100}$.

It has been checked on a most probable $\mathrm{d} E / \mathrm{d} x$ measurement [19] that the standard deviation of such distributions varies as $1 / \sqrt{ } m$. The reference was in this case the standard deviation corresponding to 10 cells of $40 \mathrm{~mm}$.

The following comments can be made about fig. 17:

(a) From the primary cluster curve $\left(\rho=0, \sigma_{0 \text { diff }}=\right.$ 0 ) the gas layer needed to separate proton from kaon within two-standard deviations at $p=10 \mathrm{GeV} / c$ is drastically reduced to $30 \mathrm{~cm}$. However, because of the faster Fermi saturation of the primary counting (fig. 8), the number of cells increases steeper with $\gamma$ than in a $\mathrm{d} E / \mathrm{d} x$ method. $150 \mathrm{~cm}$ of gas are needed at $p=40 \mathrm{GeV} / c$ and 500 at $p=80 \mathrm{GeV} / c$. This predicted behaviour emphasizes the necessity of having a best experimental knowledge of the relativistic rise of the number of primaries before any practical large size application.

(b) The influence of the detector resolution (at

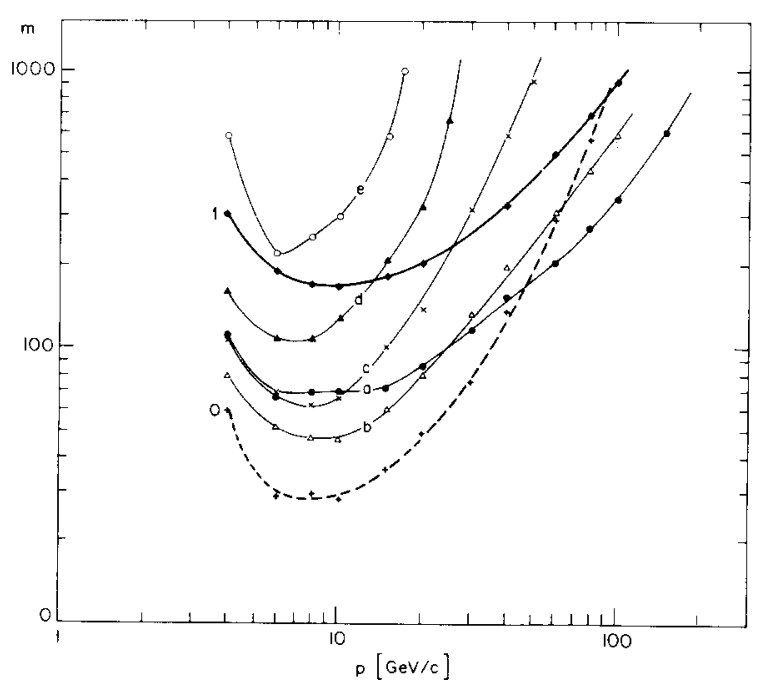

Fig. 17. Length of a detector necessary to achieve a separation proton-kaon with a two-standard deviation resolution. $m=$ number of $10 \mathrm{~mm}$ argon layer. Curve 0: primary cluster counting $\left(\sigma_{0}\right.$ diff $=0$ and $\left.\rho=0\right)$. Curve $1: \mathrm{d} E / \mathrm{d} x$ measurement in most probable value (taken as the mean of the $50 \%$ smallest of a measured sample). Curves a, b, c, d, e, respectively for $\rho=20,50,100,150,200 \mu \mathrm{m}$ and $\tau_{0 \text { diff }}=200 \mu \mathrm{m}$. $\left.\sigma_{0 \text { diff }}=200 \mu \mathrm{m}\right)$ is to increase the number of cells needed. As already pointed out, one observes that this number is minimum at $\rho=50 \mu \mathrm{m}$ at the low momentum range. In fact, the curve (a) $(\rho=20 \mu \mathrm{m})$ has a variation comparable to the curve 1 corresponding to a $\mathrm{d} E / \mathrm{d} x$ sampling: this means that, at very good resolution, a Landau or $\mathrm{d} E / \mathrm{d} x$-like behaviour is still apparent with the advantage of $m$ rising slowly at higher momenta. For higher values of $\rho$ the minimum number of cells increases rapidly to become comparable to a $\mathrm{d} E / \mathrm{d} x$ length of gas $(\rho \sim 150-$ $200 \mu \mathrm{m}$ ). The curves start rising very early, demanding for very large values of $m$, already at momenta $<20 \mathrm{GeV} / c$.

The same calculation of a detector length can be performed with the clusters counted only among selected events, for example those having a total number of electrons smaller than the most probable $N_{t}^{p p}$. A slight improvement is observed at very good resolution $\rho=20 \mu \mathrm{m}$ but results become identical at higher $\rho$. The gain obtained by the best counting made in selected layers is balanced by the increase in gas length necessary to select these events.

\section{Comparison with experiment and extension to gas mixtures}

We shall first compare the recent results obtained with a TEC [4] to the ELD simulation. The measured relativistic rise, $12 \%$, is compatible with the prediction, (fig. 11c) if the experimental resolution lies around $120 \mu \mathrm{m}$ : this is what is likely to be expected from the characteristics of the set up used. But, the number of clusters predicted at this resolution (fig. 11a) is found larger than the measurement, $\sim 20$ instead of 12.3. A tentative explanation to these features might be found in the difficulty of detecting a single electron in part II of the TEC (fig. 1): this should not perturb the relative measurement of the relativistic rise while the number of counts might be affected.

About the detection of single electron we shall recall two possible sources of problems, relative or not to ref. 4.

(1) The fluctuation of the size of an avalanche originated from one or few electrons is very large (fig. 18), particularly in the case of amplification around a wire of very small diameter $[18,20,21]$. This is apparent in relation (14) which applies to a primary counting, as the successive pulses are well resolved; $n_{i}$ 


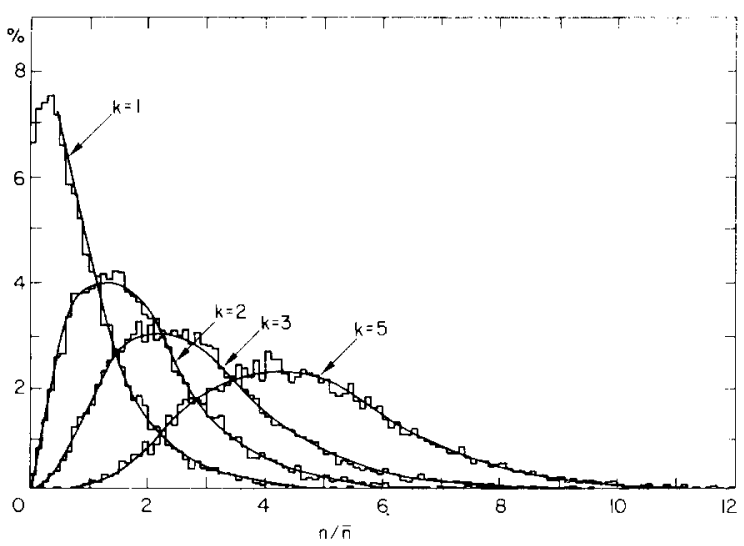

Fig. 18. Probabilities of obtaining $n$ secondaries from $\mathbf{n}$ primary electrons.

has to be taken small, i.e. equal to one in $80 \%$ of the pulses.

(2) A too high space charge around the wire can also reduce or cancel the amplification of electrons when they drift orthogonally to the sense wire and avalanche at the same place sequentially in time [9].

We shall now outline the problems connected with gas mixtures. The prediction of the number of primary clusters deposited in pure argon is certainly affected by its necessary admixture with a second or even third molecular constituent. In ELD model, we have not taken into account the excitation occurring below $I_{0}=15.7 \mathrm{eV} \mathrm{[23]} \mathrm{as} \mathrm{they} \mathrm{do} \mathrm{not} \mathrm{produce} \mathrm{elec-}$ trons in pure argon *. This is not the case in a mixture according to mechanisms such as

$\mathrm{Ar}^{*}+\mathrm{B} \rightarrow \mathrm{Ar}+\mathrm{B}^{+}+\mathrm{e}^{-}$,

or

$\mathrm{Ar}^{*} \rightarrow \mathrm{Ar}+h \nu, h \nu+\mathrm{B} \rightarrow \mathrm{B}^{+}+\mathrm{e}^{-}$,

where $\mathrm{Ar}^{*}$ is an excited state of argon and $\mathrm{B}$ a constituent with its first ionizing potential smaller than $15.7 \mathrm{eV}$. These processes should increase the number of primaries for argon. It is evident that the complexity of the simulation will rapidly grow for gas mixtures involving heavy organic molecules with low first ionization potential, such as isobutane, methylal. However, the case of argon-methane mixtures is simpler as this potential is higher in methane than the ${ }^{3} \mathrm{P}_{1}{ }^{1} \mathrm{P}_{1}$ lines in argon. That implies [15b] a very

\footnotetext{
* On the contrary, all possible ionizations originated from an excitation process of energy larger than $I_{0}$ are included in $\sigma_{\gamma}(E)$ (fig. 22). For example, the bump preceding the $\mathrm{L}$ edge is due to excitation processes.
}

small probability of argon de-excitation followed by an ionization (0.04), while this probability rises, for example, to 0.76 in an argon-acetylene mixture. This behaviour can also be seen in the fact that the Fano factor of argon is nearly unaffected by a small addition of methane but drops down to 0.07 in the case of acetylene.

In order to overcome these difficulties, one can think of getting experimentally the CSD, which includes the most difficult part of the simulation, that is the secondary mechanisms and the low energy electron multiplication. This distribution could be obtained by measuring, in a gas mixture, the number of electrons released in a given transfer produced by low energy photons in the range 10 to $1000 \mathrm{eV}$ which are now available around a synchroton radiation facility [23]. The two input data sets in the simulation would now be the CSD and the photo absorbtion cross section, obtainable by the same technique.

\section{Conclusions}

The combined effects of the longitudinal diffusion and the detector resolution on a primary cluster counting in argon, under the conditions of sect. 2 , permit a significant measurement of the pattern of deposited clusters for a very small range of resolution around $50 \mu \mathrm{m}$. A finer resolution, $\rho \simeq 20 \mu \mathrm{m}$, will reveal a Landau behaviour while for a coarser one, $\rho \geq 100 \mu \mathrm{m}$, which is of the same order of magnitude than the diffusion effect, the measured pattern of clusters is less and less correlated to the initial one. This latter situation also implies a loss of counts which makes particle identification more difficult, even if the energy resolution remains drastically improved, $40 \%$ fwhm in $10 \mathrm{~mm}$ or argon instead of the $100 \%$ of a Landau spectrum. In spite of a reduced relativistic rise ratio (1.32) a cluster counting technique will shorten by a factor of two the gas path necessary for a $\mathrm{p}-\mathrm{k}$ separation up to $15 \mathrm{GeV} / c$ with an electronic separation of two clusters of $\sim 10 \mathrm{~ns}$ $(\rho \cong 100 \mu \mathrm{m})$. However, this factor should be taken as an optimum value, considering other technical problems not included in our simple definition of the dead time $\tau$, such as pulse height dynamics in the detection chamber. Finally, the fact that the Fermi saturation occurs earlier for the primary deposition $(\gamma \cong 200)$ makes the method less promising at higher momentum.

Our calculations on the relativistic rise of the 
primary clusters are compatible with the experimental results of ref. 4 within the uncertainty due to the different gas mixture.

We are indebted to Drs. J.H. Cobb, D. Drijard, I. Lehraus and A.H. Walenta for valuable discussions and comments.

We thank Dr. P.G. Innocenti for his critical reading of the manuscript and his constructive remarks.

\section{Appendix A}

\section{$x$ distribution of primary collisions}

The number of primary interactions follows a Poisson-like statistics, i.e. if $N$ is the average number of primary interactions, the probability of having $k$ in one event is

$p_{k}^{N}=\frac{N^{k}}{k !} \mathrm{e}^{-N}$.

The $x$ distribution of the $j$ th primary interaction in the gap is [24]

$A_{j}^{N}(x)=\frac{x^{j-1}}{(j-1) !} N^{j} \mathrm{e}^{-N x}$,

then, the probability to find the $j$ th primary interaction between $a$ and $b$ is

$\mathcal{P}(a \leqslant x \leqslant b)=\int_{a}^{b} A_{j}^{N}(x) \mathrm{d} x$.

These results will be compared to those which are obtained by a Monte Carlo method (fig. 19). If $\lambda$ is the mean free path of the charged particle, the probability that the collision occurs between $l$ and $l+\mathrm{d} l$ is $f(l)=-\lambda^{-1} \exp (-l / \lambda)$. In order to pick $l$ randomly but weighted by $f(l)$, we use $l=-\lambda \log (\xi)$, where $\xi$ is a random number uniformly distributed between 0 and 1.

\section{Appendix B}

\section{Energy loss and cluster size distribution in argon}

We have concentrated on examining the collisions of relativistic charged particles traversing a thin layer of gas in as complete a way as possible. Namely, we

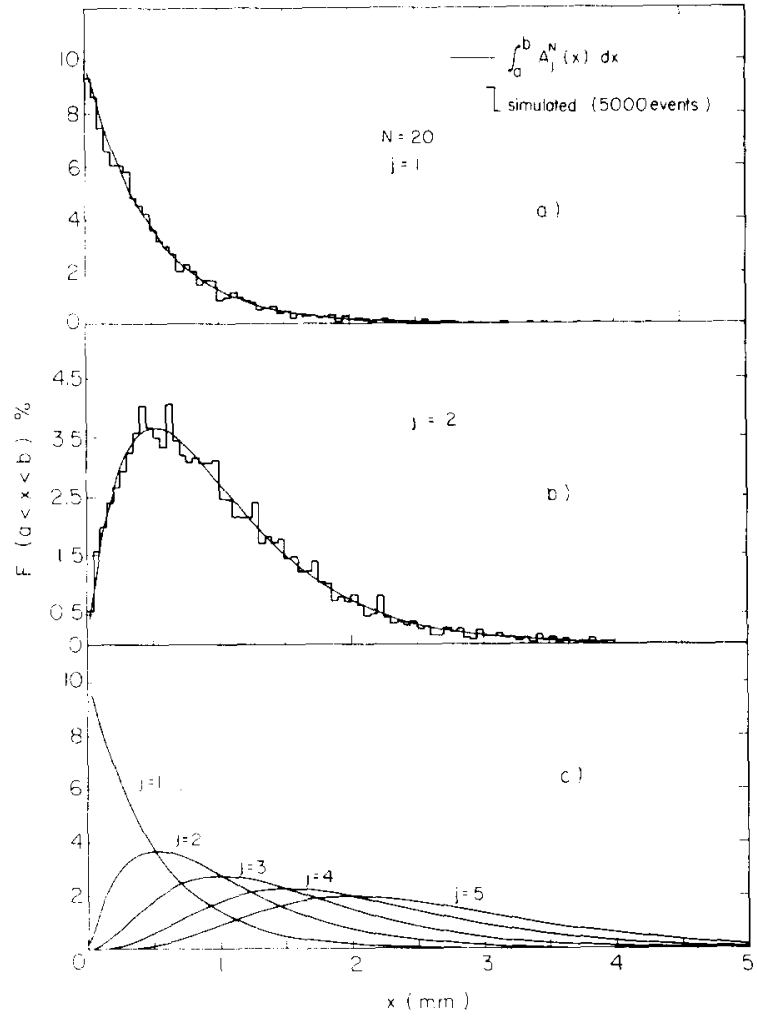

Fig. 19. $x$ distributions of the $j$ th primary interaction $(j=1-$ 5) obtained by a Monte Carlo method (a) and (b). The dotted lines are the calculated $x$ distributions with $N=20$ interactions per centimeter.

have tried to know the number of created secondary electrons for a given transfer.

The flow chart which describes the different steps of the simulation is shown in fig. 20.

Note that the only experimental data necessary from the beginning are the following:

$-\sigma_{\gamma}(E)$ photoabsorption cross section,

- decay processes of Ar for a given transfer.

\section{Appendix C}

\section{Energy transfer spectrum for individual collisions}

The energy loss for relativistic charged particles in a thin gas layer $(<10 \mathrm{~cm})$ is the sum of a large number of small energy transfers (less than $50 \mathrm{eV}$ ) and a small number of big transfers (several hundred $\mathrm{eV}$ to several $\mathrm{keV}$ ).

In the range of the great transfers (some $\mathrm{keV}$ ) the gas electrons may be considered as free. In such a 


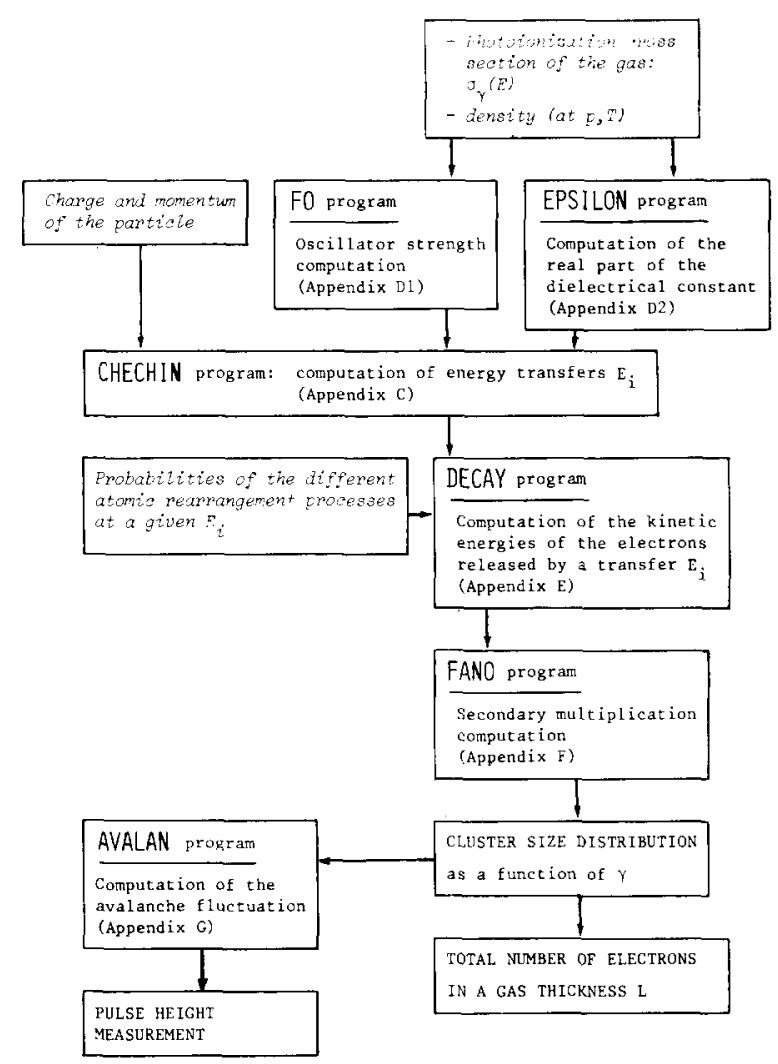

Fig. 20. Simulation flow chart of the cluster size distribution and energy loss.

case the differential cross section of inelastic collisions or $\mathrm{d}^{2} N / \mathrm{d} x \mathrm{~d} E$, the number of collisions per unit length with energy transfers in the range $E$ to $E+\mathrm{d} E$ is given by the Rutherford scattering cross section

$\frac{\mathrm{d}^{2} N}{\mathrm{~d} x \mathrm{~d} E}=\frac{A}{\beta^{2}} \frac{1}{E^{2}} \mathrm{MeV}^{-1} \mathrm{~cm}^{-1}$,

where $A=0.1536 z^{2}\left(Z / A_{0}\right) \rho \mathrm{MeV} / \mathrm{cm}, Z, A_{0}$ and $\rho$ are atomic number, mass number and density of the medium; $\beta$ and $z e$ respectively velocity and charge of the incident particle.

In connection with the calculation of the relativistic rise of bubble density in bubble chambers Chechin et al. [10(a)] proposed formulas that enable to calculate the primary specific ionizations $n_{1}$ with a great degree of accuracy

$$
\begin{gathered}
\frac{\mathrm{d}^{2} N}{\mathrm{~d} x \mathrm{~d} E}=\frac{A}{\beta^{2}}\left\{\frac{f(E)}{E}\left[\log \frac{2 m_{\mathrm{e}} c^{2} \beta^{2}}{\left(1-\beta^{2}\right) E}-\beta^{2}\right]\right. \\
\left.+\frac{1}{E^{2}} \int_{0}^{E} f(E) \mathrm{d} E-\Delta^{\prime}(\beta, \epsilon)\right\},
\end{gathered}
$$

where $f(E)$ are the distributed oscillator strengths (appendix D) and $\Delta^{\prime}$ the density effect correction (for gases at NTP $\Delta^{\prime} \sim 0$ up to $\gamma \sim 20-30$ )

$$
\begin{aligned}
-\Delta^{\prime} & =\frac{f(E)}{E}\left[\log \frac{\left(1-\beta^{2}\right)}{\left|1-\beta^{2} \epsilon\right|}+\beta^{2}(1-\operatorname{Re} \epsilon)\right] \\
+ & \frac{2}{\left(\hbar \nu_{\mathrm{p}}\right)^{2}}\left(\beta^{2}-\frac{\operatorname{Re} \epsilon}{|\epsilon|^{2}}\right),
\end{aligned}
$$

$\nu_{\mathrm{p}}=\left(4 \pi e^{2} n / m_{\mathrm{e}}\right)^{1 / 2}, n$ the number of electrons per $\mathrm{cm}^{3}$ of matter and $\epsilon$ the complex dielectric permeability (appendix D). Therefore

$n_{1}=(\mathrm{d} N / \mathrm{d} x)_{I_{0}<E<E_{\max }}$,

where $I_{0}$ is the first ionization potential and $E_{\max }$ is the kinematically maximum energy transfer. The number of collisions per unit length inducing an energy transfer greater than $E$ (fig. 21) is

$$
\begin{aligned}
& \left(\frac{\mathrm{d} N}{\mathrm{~d} x}\right)_{>E}=\int_{E}^{E_{\max }}\left(\frac{\mathrm{d}^{2} N}{\mathrm{~d} x \mathrm{~d} E}\right) \mathrm{d} E \\
& =\frac{A}{\beta^{2}}\left\{\int _ { E } ^ { E _ { \operatorname { m a x } } } \frac { f ( E ) } { E } \left[\log \frac{2 m_{\mathrm{e}} c^{2} \beta^{2}}{\left(1-\beta^{2}\right) E}-\beta^{2}+1\right.\right. \\
& \left.\quad-\frac{E}{E_{\max }}\right] \mathrm{d} E \\
& \left.\quad+\left(\frac{1}{E}-\frac{1}{E_{\max }}\right) \int_{0}^{E} f(E) \mathrm{d} E-\int_{E}^{E_{\max }} \Delta^{\prime}(\epsilon) \mathrm{d} E\right\} .
\end{aligned}
$$

The last term in eq. (6) is the contribution of Cheren-

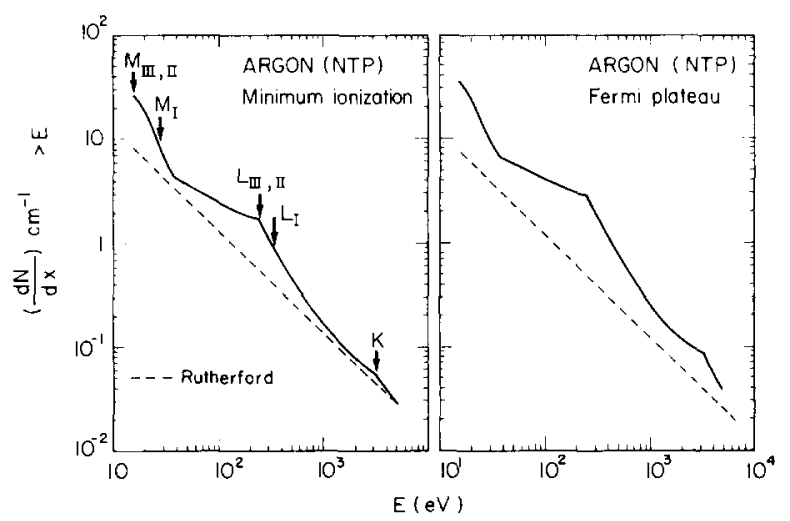

Fig. 21. Number of collisions per unit length including an energy transfer greater than $E$. The arrows show the absorption edges of the various atomic shells: (a) minimum ionization and (b) Fermi plateau. 
kov radiation which has to be taken into account only when the condition $\beta^{2} . \operatorname{Re}(\epsilon)>1$ is satisfied for photon energies greater than $I_{0}$. In pure argon this contribution will amount to $1-2 \%$ (appendix D).

\section{Appendix D}

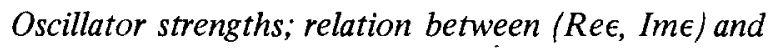
$\sigma_{\mathcal{\gamma}}(E)$

\section{1) Oscillator strengths.}

The oscillator strengths $f(E)$ satisfy the ThomasReich-Kuhn sum rule [25]

$\sum f=\sum_{s}^{\text {dis }} f_{s}+\int_{I_{0}}^{\infty} f_{\mathrm{c}}(E) \mathrm{d} E=Z$,

where $Z$ is the atomic number. We calculate $f_{\mathrm{c}}(E) *$ from experimental photo-absorption cross section $\sigma_{\gamma}(E)$ (fig. 22)

$f_{\mathrm{c}}(E)=\frac{\sigma_{\gamma}(E)}{2 \pi^{2} r_{0} \hbar c Z}=\frac{\sigma_{\gamma}(E)}{1.097 \times 10^{-16} Z}$,

where $E$ is the photon energy and $r_{0}=e^{2} \mid m c^{2}$. The parametrization of $\sigma_{\gamma}(E)$ can be written as:

$E>3206 \mathrm{eV}: \quad \sigma_{\gamma}(E)=0.1\left(\frac{3206}{\epsilon}\right)^{2.75} \mathrm{Mb}$;

$248<E<3206: \quad \sigma_{\gamma}(E)=4.51\left(\frac{248}{\epsilon}\right)^{2.29} \mathrm{Mb} ;$

$15.75<E<248$ : (see data of ref. 11 and table 4 in appendix E).

There are few theories and accurate experimental results concerning $f_{s}$, the oscillator strengths of the discrete transitions corresponding to the excited atom. We used the values of table 3 [26]:

Samson believes that is is not too unreasonable to expect that the total contribution from the remaining discrete lines could be of the order of one [27].

For argon, the individual contributions to $f_{\mathrm{c}}$ are as follows:

threshold to L edge: 0.3947 ,

L edge to K edge: 0.4228 ,

K edge to $100 \mathrm{keV}$ : 0.0925 ,

total:

0.91 .

* The oscillator strengths were normalized to unity in the formulas of Appendix C.

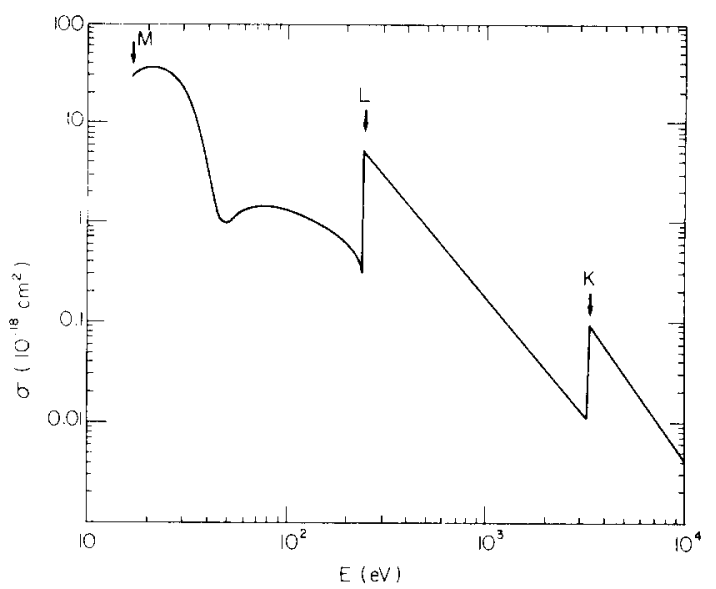

Fig. 22. Parametrization of the photoabsorption cross section $\sigma_{\gamma}(E)$ used in the simulation.

$\sum=\sum_{s} f_{s}+\int_{I_{0}}^{\infty} f_{\mathrm{c}}(E) \mathrm{d} E=0.94$.

The values of $f(E)$ were normalized in the calculations in such a way as they satisfy the sum rule rigorously.

An other useful quantity for the test of the calculation of $f(\epsilon)$ is $I$, the mean ionization potential defined by

$\ln I=\int_{0}^{\infty} \ln (E) f(E) \mathrm{d} E$.

For argon, the calculated value of $I$ is $180 \mathrm{eV}$. It must be compared to the experimental value $200 \mathrm{eV}$ which is usually given in the literature [28].

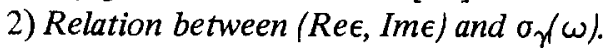

Table 3

Oscillator strengths

\begin{tabular}{lc}
\hline$\AA$ & $\mathrm{f}^{\text {a) }}$ values $\left(\times 10^{3}\right)$ \\
\hline 1066.86 & $59 \pm 3$ \\
1048.22 & $228 \pm 21$ \\
877.95 & $28 \pm 3$ \\
869.75 & $13 \pm 3$ \\
876.06 & $93 \pm 6$ \\
866.81 & $107 \pm 15$ \\
& Total: 0.528
\end{tabular}

a) Values not normalized to unity. 


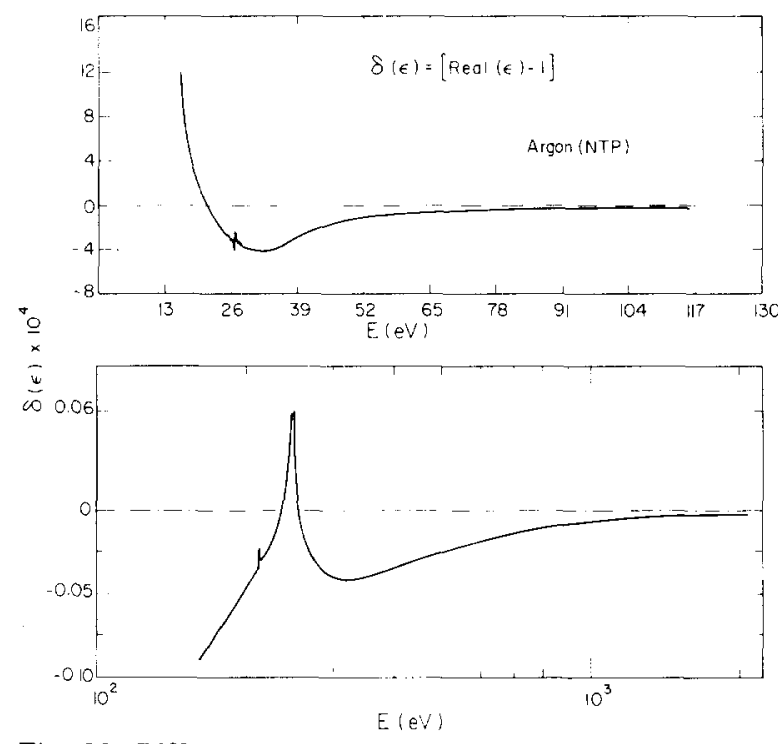

Fig. 23. Difference between the real part of the complex refractive index and unity versus energy.

The complex refractive index is written as [29]

$\epsilon=(n+\mathrm{i} k)^{2}=n^{2}-k^{2}+2 n \mathrm{i} k$,

where $k$ is known as the absorption coefficient of electromagnetic radiation. For gases $n \simeq 1$ and $k \ll 1$ thus

$\operatorname{Re}(\epsilon) \sim n^{2}$

$\operatorname{Im}(\epsilon) \sim 2 k=\frac{c}{\omega} \eta \sigma_{\gamma}(\omega)$,

$\chi$ is the number of atoms $/ \mathrm{cm}^{3}$ and $\sigma_{\gamma}$ the phiotoabsorption cross section.

\section{Calculation of $\operatorname{Re}(\epsilon)$}

In this paper we calculate the real part of $\epsilon$ of argon for a wide range of energies by using the Kramers-Kronig dispersion relations [29]

$\operatorname{Re} \epsilon(\omega)=1+\frac{2}{\pi} \mathcal{P} \int_{0}^{\infty} \frac{\omega^{\prime} \operatorname{Im} \epsilon\left(\omega^{\prime}\right)}{\omega^{\prime 2}-\omega^{2}} \mathrm{~d} \omega^{\prime}$,

where $\mathcal{P}$ denotes the Cauchy principal value. Thus, with eq. (11) [30]

$$
\begin{aligned}
\delta(\epsilon)=\operatorname{Re}[\epsilon(E)-1] & \\
= & \mathcal{R}\left[A \sum_{n} \frac{f_{0 n}}{E_{0 n}^{\prime 2}-E^{2}}\right. \\
& \left.+B \mathcal{P} \int_{I_{0}}^{\infty} \frac{\sigma_{\gamma}\left(E^{\prime}\right) \mathrm{d} E^{\prime}}{E^{\prime 2}-E^{2}}\right],
\end{aligned}
$$

the constants $A$ and $B$ are

$$
\begin{aligned}
A & =4 \pi \frac{e^{2}}{m c^{2}}(\hbar c)^{2}\left(\mathrm{eV}^{2} \cdot \mathrm{cm}^{3}\right) ; \\
B & =\frac{2}{\pi} \hbar c(\mathrm{eV} \cdot \mathrm{cm}) .
\end{aligned}
$$

$\delta(\epsilon)$ is represented in fig. 23.

\section{Appendix E}

Multiple ionization of argon as function of the energy transfer

Argon.

The multiple ionization and decay processes of $\mathrm{Ar}$ for a range of energy transfers up to $400 \mathrm{eV}$ has been studied in detail by Van der Wiel et al. [31]. Taking these results into consideration, we will only retain in this paper the most probable decay processes. Table 4 gives the energies corresponding to the different shells of the argon atom.

(a) Ionization in the $M$ shell

The distribution of oscillator strengths for $\mathrm{Ar} \mathrm{M}$ shell ionization leading to the charge states one to three can be found in fig. 3 of ref. 31 . On the smooth background of direct ionization, two electron excited states of type $3 \mathrm{~s}^{1} 3 \mathrm{p}^{5} n n^{\prime} l$ are superposed. These excited states decay to a single or double charged final state $\left(\mathrm{Ar}^{2+} / \mathrm{Ar}^{1+} \sim 0.15\right.$ for energy losses up to $\sim 70 \mathrm{eV}$ ).

Neglecting the decay into $\mathrm{Ar}^{2+}$, an energy transfer $E$ smaller than $E_{\mathrm{L}_{2,3}}$ shell is equivalent, for us, to an electron with a kinetic energy $K$

$K=E-E_{\mathrm{M}}$.

\section{(b) Ionization in the $L$ shell}

The oscillator strength spectra can be found in fig. 4 of ref. 31. At energy transfer, a few eV above the $L_{2,3}$ thresholds, the highest contribution comes

Table 4

Energies for different shells of argon

\begin{tabular}{lrll}
\hline & eV & & eV \\
\hline$E_{\mathrm{K}}$ & 3206.0 & $E_{\mathrm{M}_{1}}$ & 29.2 \\
$E_{\mathrm{L}_{1}}$ & 326.3 & $E_{\mathrm{M}_{2}}$ & 15.9 \\
$E_{\mathrm{L}_{2}}$ & 250.7 & $E_{\mathrm{M}_{3}}$ & 15.7 \\
$E_{\mathrm{L}_{3}}$ & 248.6 & & \\
\hline
\end{tabular}


from the ordinary Auger process

$$
\begin{aligned}
2 \mathrm{p}^{5} 3 \mathrm{p}^{6} & \rightarrow 2 \mathrm{p}^{6} 3 \mathrm{p}^{4}+\mathrm{e}^{-}, \\
2 \mathrm{p}^{5} & \rightarrow \mathrm{Ar}^{2+},
\end{aligned}
$$

A certain probability exists for the excess energy to be used in the ejection of two $\left(\rightarrow \mathrm{Ar}^{3+}\right)$ or three $\left(\rightarrow \mathrm{Ar}^{4+}\right)$ electrons.

At the thresholds for simultaneous ionization in inner and outer shells, corresponding to the configuration $2 \mathrm{p}^{5} 3 \mathrm{p}^{5}(\sim 280 \mathrm{eV})$ and $2 \mathrm{p}^{5} 3 \mathrm{~s}^{1}(\sim 300 \mathrm{eV})$, the final product - with a probability of $\sim 0.2$ - is $\mathrm{Ar}^{3+}$.

When $L_{1}$ vacancy has been created, the charged final state - with a probability of $\sim 0.35-$ is $\mathrm{Ar}^{3+}$. In this case, two steps must be considered:

(1) a Coster-Kronig transition $2 s^{1} 2 p^{6} \quad 3 p^{6} \rightarrow$ $2 s^{2} 2 p^{5} 3 p^{5}+e$,

(2) the Auger process descibed before.

The Auger line energy of eq. (13) is $\sim 200 \mathrm{eV}[12 \mathrm{~b}]$, the Coster-Kronig line energy is $\sim 50 \mathrm{eV} \mathrm{[32].}$

At still higher energy losses an ionization in the $2 \mathrm{~s}$ shell may be accompanied by additional ionization in the outer shell $-2 s^{1} 3 p^{5}$ or $2 s^{1} 3 s^{1}$. Table 5 gives a summary.

(c) Ionization in the $K$ shell

An initial vacancy in the $K$ shell may be filled either by the emission of $\mathrm{K}$ series $\mathrm{X}$ radiation (fluorescence yield $\omega_{K}$, for argon $\omega_{K} \sim 0.12$ ) or of $K$ Auger electrons. The considered processes are $\mathrm{K}$ and

Table 5

\begin{tabular}{|c|c|c|c|}
\hline $\begin{array}{l}\text { Energy } \\
\text { transfer } E\end{array}$ & $\begin{array}{l}\text { Proba- } \\
\text { bility }\end{array}$ & $n b$ of $e^{-}$ & $\begin{array}{l}\text { Kinetic energy } \\
(\mathrm{eV})\end{array}$ \\
\hline$E_{\mathrm{L}_{2,3}}<E<280$ & 1 & 2 & $\begin{array}{l}K_{1}=E-E_{\mathrm{L}_{2,3}} \\
K_{2}=200 \mathrm{eV}\end{array}$ \\
\hline $280<E<E_{\mathbf{L}_{1}}$ & $\begin{array}{l}0.8 \\
0.2\end{array}$ & 2 & $\begin{array}{l}K_{1}=E-E_{\mathrm{L}_{2,3}} \\
K_{2}=200 \mathrm{eV} \\
K_{1}+K_{2}=E- \\
E_{\mathrm{L}}-E_{\mathrm{M}} \\
K_{3}=200 \mathrm{eV}\end{array}$ \\
\hline$E>E_{\mathrm{L}_{1}}$ & $\begin{array}{l}0.65 \\
0.35\end{array}$ & $\begin{array}{l}2 \\
3\end{array}$ & $\begin{array}{l}K_{1}=E-E_{\mathrm{L}_{2,3}} \\
K_{2} \text { a) }=200 \mathrm{eV} \\
K_{1}=E-E_{\mathrm{L}_{1}} \\
K_{2}=50 \mathrm{eV} \\
K_{3}=200 \mathrm{eV}\end{array}$ \\
\hline
\end{tabular}

Summary of transitions with corresponding energy

a) The contribution from decay of the $\mathrm{L}_{1}$ state to $\mathrm{Ar}^{2+}$ via direct Auger transition is small.
L Auger electrons [12b]

$E_{\mathrm{K}}-E_{\mathrm{L} 3} E_{\mathrm{L} 3}, \quad E=2670 \mathrm{eV}$;

$E_{\mathrm{L} 3}-E_{\mathrm{M} 3} E_{\mathrm{M} 3}, \quad E=200 \mathrm{eV}$.

\section{Appendix F}

\section{Low energy electrons in argon}

The collisions of electrons with atoms and molecules can be divided into three classes: elastic scattering, impact ionization and impact excitation. We will describe the evaluation of inelastic cross sections in argon, in view of a Monte Carlo calculation.

1) Impact ionization

To perform the energy loss, the total ionization cross section $\sigma_{i}$ (fig. 5.26 in ref. 13) and the differential ionization cross section $(\mathrm{d} \sigma / \mathrm{d} K)_{\mathrm{i}}$, i.e. the differential cross section for an ionizing collision in which the energy of the ejected electrons lies between $K$ and $K+\mathrm{d} K$, must be known

$\sigma_{i}=\iint_{\epsilon} I\left(K_{\mathbf{p}}, K, \theta\right) \mathrm{d} \epsilon \mathrm{d} \Omega$,

$\left(\frac{\mathrm{d} \sigma}{\mathrm{d} K}\right)=\int_{\Omega} I\left(K_{\mathrm{p}}, K, \theta\right) \mathrm{d} \Omega$,

where $I\left(K_{\mathrm{p}}, K, \theta\right)$ is the double differential cross section, $K_{\mathrm{p}}$ the energy of the incident electron and $\theta$ the scattered angle. The upper limit for integration over $K$ is $\frac{1}{2}\left(K_{\mathrm{p}}-I\right)$ and $I$ the ionization potential. Measurements of $I\left(K_{\mathrm{p}}, K, \theta\right)$ and $(\mathrm{d} \sigma / \mathrm{d} K)$ have been made by Opal et al. $[33,34]$.

For ejected electron energies much less than $I$, the cross section approaches a constant value, whereas for sufficiently high energies the cross section falls off as $K^{-2}$.

In addition (especially for heavy noble gases) one finds excitation $E_{\text {exc }}$ of neutral argon singlet and triplet states having the configuration $3 \mathrm{~s}^{1} 3 \mathrm{p}^{6} \mathrm{nl}$. These states autoionize [35] to either the ${ }^{2} P_{1 / 2}$ or ${ }^{2} P_{3 / 2}$ ground state of $\mathrm{Ar}^{+}$

$$
3 \mathrm{~s}^{2} 3 \mathrm{p}^{6} \rightarrow 3 \mathrm{~s} 3 \mathrm{p}^{5} n l^{\uparrow^{3 \mathrm{~s}^{2} 3 \mathrm{p}^{5}\left({ }^{2} P_{1 / 2}\right)+\mathrm{e}^{-},}} \stackrel{\downarrow}{\downarrow \mathrm{s}^{2} 3 \mathrm{p}^{5}\left({ }^{2} P_{3 / 2}\right)+\mathrm{e}^{-} .}
$$

The energy of the ejected electrons

$K=E_{\mathrm{exc}}-E_{\mathrm{M} 2,3}$ 
is independent of the energy of the primary electrons. These states are omitted in the calculation.

The relations which will be used for $\mathrm{K}$ calculations are the following:

$-K_{\mathrm{p}} \leqslant 100 \mathrm{eV}$ :

$$
K=\xi K_{\max }, \quad\left(\left(\frac{\mathrm{d} \sigma}{\mathrm{d} K}\right)_{\mathrm{i}}=\text { constant }\right) ;
$$

$-K_{\mathrm{p}}>100 \mathrm{eV}:$

$$
\begin{aligned}
& K=\frac{K_{\max }}{K_{\max }-\xi\left(K_{\max }-1\right)}, \\
& \left(\left(\frac{\mathrm{d} \sigma}{\mathrm{d} K}\right)_{\mathrm{i}} \sim \frac{1}{K^{2}}\right) .
\end{aligned}
$$

$K_{\max }=\frac{1}{2}\left(K_{\mathrm{p}}-I\right), \xi$ is a random number uniformly distributed between 0 and 1 .

\section{2) Impact excitation.}

This process is of great importance, because it is mainly responsible for the increase of $w$, i.e. the average energy loss per ion pair formed, when $K_{\mathrm{p}}$ decreases [36].

The total excitation cross section $\sigma_{\text {exc }}$ is defined by

$$
\sigma_{\mathrm{exc}}=\sum_{j} \sigma_{\mathrm{exc}}^{j},
$$

where $\sigma_{\text {exc }}^{j}$ is the excitation of the $j$ th level. The available theoretical and experimental information on excitation of rare gases atoms by electron impact has been summarized and discussed by Moisewitsch and Smith (1968) [37]. For argon we used the data of Zapesochnyi and Feltsan (1966) [38]. The sum of the maximum value of $\sigma_{\text {exc }}^{j}$ is $\sim 10 \times 10^{-17} \mathrm{~cm}^{2}$, thus for $K_{\mathrm{p}} \sim 20-35 \mathrm{eV}$ one obtains the ratio

$\sigma_{\text {exc }} / \sigma_{\mathrm{i}} \sim 1$.

For $K_{\mathrm{p}}>50 \mathrm{eV}$, the ratio $\sigma_{\text {exc }} / \sigma_{\mathrm{i}}$ is taken constant and equal to 0.4 [14b]. The mean excitation energy necessary to perform the energy losses due to impact excitation is calculated according to

$$
\widetilde{K_{\text {exc }}}=\frac{\sum_{j} \sigma_{\mathrm{exc}}^{j} \cdot K_{\mathrm{exc}}}{\sum_{j} \sigma_{\mathrm{exc}}^{j}} \sim 13 \mathrm{eV},
$$

$\widetilde{E_{\text {exc }}}$ is constant with respect of $K_{\mathrm{p}}$.

3) Calculation procedure.

As the angular information is unimportant for this purpose, the elastic scattering cross section is not taken into account.
In the case of an excitation, the energy loss is equal to $\overline{E_{\text {exc }}}$. If a ionization event occurs, two possibilities must be distinguished with respect to the energy of the produced secondary electron. The kinetic energy is: (a) lower than $I_{0}$ and (b) exceeds $I_{0}$. In this last case the produced electron is handled in the same way as the starting electron.

4) Results.

In order to be reassured about the validity of our calculation model, results on such important quantities as $w$ and $F$ (Fano factor) will be compared with experimental data.

The mean work to create a ion-pair is defined by

$w=E_{0} / N_{\mathrm{i}}$

where $E_{0}$ is the energy of the charged particle (totally absorbed) and $N_{\mathrm{i}}$ is the total number of ion-pairs produced.

The statistical fluctuations of $N_{\mathrm{i}}$ can be written as follows:

$\overline{\left(N_{\mathrm{i}}-\bar{N}_{\mathrm{i}}\right)^{2}}=F \bar{N}_{\mathrm{i}}$,

here $F$ is known as the Fano factor and $\bar{N}_{\mathbf{i}}$ is an average value of $N_{\mathrm{i}}$.

The experimental value for $w$ is $26.4 \mathrm{eV} \mathrm{[13]} \mathrm{and,}$ for a gas mixture of $\mathrm{Ar}+10 \% \mathrm{CH}_{4}, \mathrm{~F}$ is equal to 0.19 [17]. Figs. 24 and 25 show the result of our model for different energies of incident electrons.
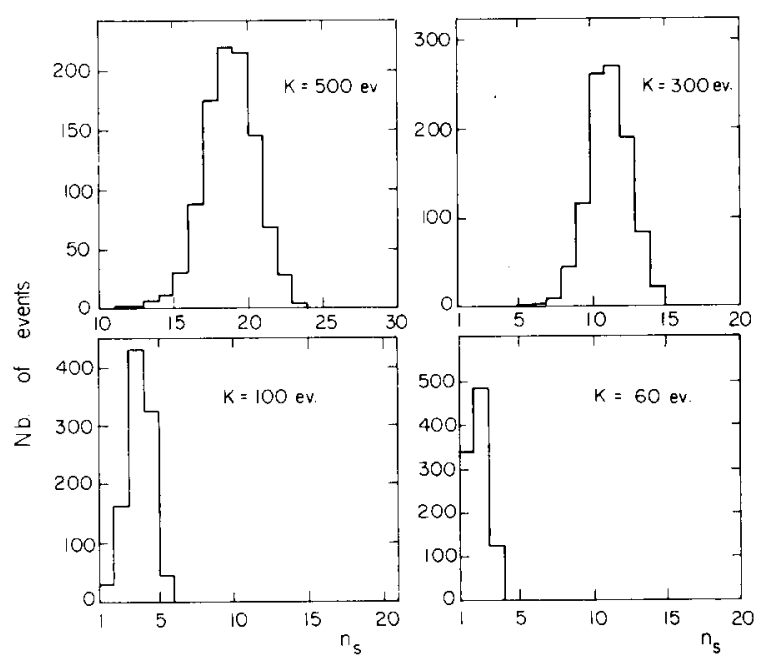

Fig. 24. Simulation of the number of ion pairs created by $50,60,100$ and $300 \mathrm{eV}$ incident electron. 


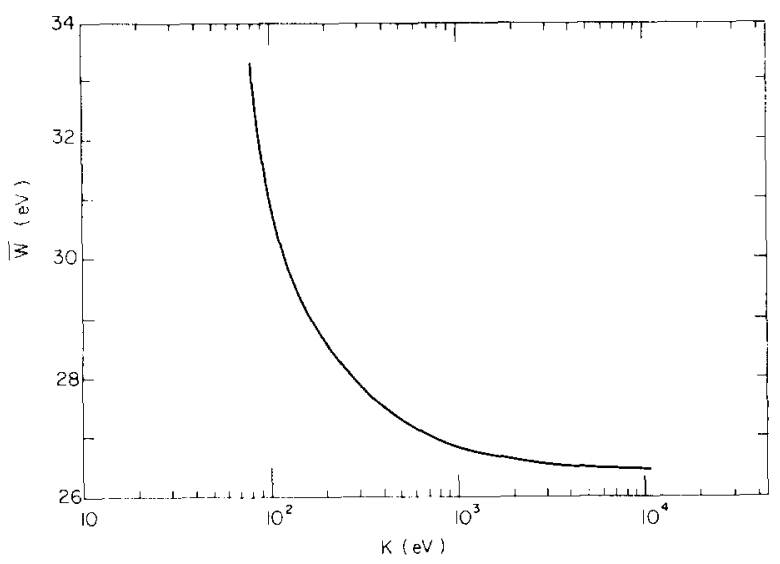

Fig. 25. Dependence of the $w$ value on electron energy.

\section{Appendix G}

\section{Avalanche fluctuations}

\section{1) Relative variance of pulse height.}

Let us call $p_{i}$ the final number of electrons into an avalanche initiated by $n_{i}$ electrons. Its fluctuation is given by [18]

$\left(\frac{\sigma_{p i}}{\bar{p}_{i}}\right)^{2}=\frac{1}{\overline{n_{i}}}\left(\frac{\sigma_{A}}{\bar{A}}\right)^{2}$

where $\bar{A}$ is the mean amplification coefficient of the detection system, and $\bar{p}_{i}=\bar{n}_{i} \bar{A} \cdot \bar{A}$ may also be considered as the number of electrons in an avalanche generated by a single electron.

An ionizing particle which deposited $N_{0}$ primaries, generates at a wire of the counter a number of electrons

$$
P=\sum_{i=1}^{N_{0}} p_{i}
$$

with

$$
\left(\frac{\sigma_{P}}{\bar{P}}\right)^{2}=\frac{1}{\sum_{i=1}^{N_{0}} n_{i}}\left(\frac{\sigma_{A}}{\bar{A}}\right)^{2}=\frac{1}{N_{\text {tot }}}\left(\frac{\sigma_{A}}{\bar{A}}\right)^{2} .
$$

Relations (15) and (16) are only valid if all the contributions of individual avalanches are summed up during a time greater than the collection time of all primaries: this is realized electronically by a convenient integrator. They imply in addition that the development of an avalanche is independent of the others, which requires a low amplification. Anyhow, due to the complexity of the phenomena involved in the avalanche, these relations have to be considered as an approximation.

2) Calculation of the relative variance of amplification factor $\sigma_{A} / \bar{A}$.

Several theoretical analyses have been made of the statistical distribution of the number of electrons in the avalanche generated in a proportional counter by a single electron; the simplest analysis [39] leads to the simple exponential law

$P(A)=\exp (-A)$,

$f=\left(\sigma_{A} / \bar{A}\right)^{2}=1$.

Curran et al. [18a], using a counter containing a mixture of argon and methane, fit the experimental data with the function

$P(A)=A^{1 / 2} \exp (-A)$,

$f=0.666$.

Alkhazov [18b] with different hypotheses on the ionization probability per unit path length versus $\xi$ ( $\xi$ the distance covered by an electron towards the

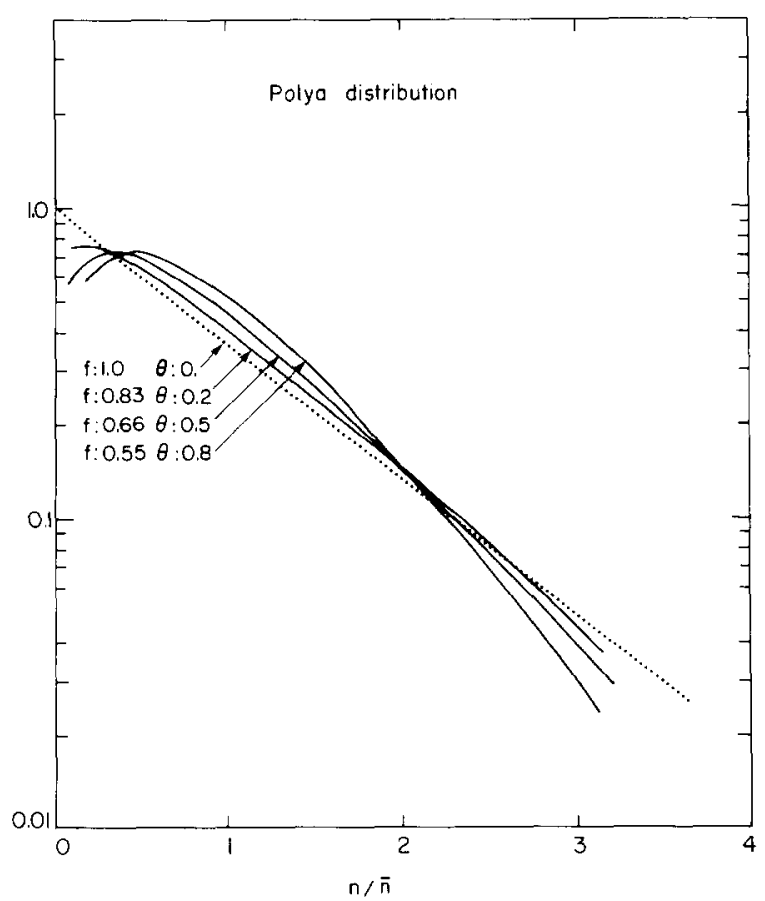

Fig. 26. Probability distribution of the Polya type for $\theta=0$, $0.2,0.5$ and 0.8 . 
anode after the last ionization) shows that the distributions in the non-uniform fields of cylindrical counters are close to those of Polya type

$P(A)=A^{\theta} \exp [-(1+\theta) A]$,

$f=\frac{1}{1+\theta}$.

Fig. 26 shows probability distributions of Polya type for different values of the parameter $\theta$. The exact physical significance of $\theta$ is not clear. Its value is subject to discussion as it depends on the detector gases and of the collection mode [20]. Byrne [21] indicates limiting values of $0.6-1.0$, the smallest values corresponding to a reduced amplification.

The Polya distribution with $\theta=0.5$ has been used in the model of the avalanche fluctuations.

\section{References}

[1] M. Aderholz, P. Lazeyras, I. Lehraus, R. Matthewson and W. Tejessy, Nucl. Instr. and Meth. 123 (1975) 237.

[2] I. Lehraus, R. Matthewson, W. Tejessy and M. Aderholz, Nucl. Instr. and Meth. 153 (1978) 347.

[3] A.H. Walenta, IEEE Trans. Nucl. Sci. NS-26 (1979) 73.

[4] P. Rehak and A.H. Walenta, BNL Report 26862.

[5] G. Schultz, Thesis (CERN).

[6] UCRL-2426, High energy particle data, vol. 2.

[7] S.V. Starodubtsev and A.M. Romanov, The passage of charged particles through matter, AEC-tr-6468 (1962).

[8] J.H. Cobb, D. Phil. Thesis (University of Oxford, 1975) available from Rutherford as HEP/T/55; J.H. Cobb, W.W.H. Allison and J.N. Bunch, Nucl. Instr. and Meth. 133 (1976) 315.

[9] H. Frehse, F. Lapique, M. Panter and F. Piuz, Nucl. Instr. and Meth. 156 (1978) 87.

[10] (a) V.A. Chechin, L.P. Kotenko, G.I. Merson and V.C. Yermilova, Nucl. Instr. and Meth. 98 (1972) 577. (b) V.C. Ermilova, L.P. Kotenko and G.I. Merzon, Nucl. Instr. and Meth. 145 (1977) 555.

[11] G.V. Marr and J.B. West, At. Data Nucl. Data Tables 18 (1976).

[12] (a) E.H.S. Burhop and W.N. Asaad, Adv. At. Molec. Phys. 8 (1972) 164.

(b) At. Data Nucl. Data Tables 20 (1977) 339.
[13] L.G. Christophorou, Atomic and molecular radiation physics (J. Wiley, New York, 1971).

[14] I.P. Zapesuchnyi and P.V. Feltsan, Opt. Spectrosc. 20 (1966) 291.

[15] (a) U. Fano, Phys. Rev. 72 (1947) 26.

(b) G.D. Alkhazov, A.P. Komar and A.A. Vorubev, Nucl. Instr. and Meth. 48 (1967) 1.

[15] M.W. Charles and B.A. Cooke, Nucl. Instr. and Meth. 61 (1968) 31.

[17] M. Kase, J. Kikuchi and T. Doke, Nucl. Instr. and Meth. 163 (1979) 289.

[18] (a) S.C. Curran, A.L. Cockroft and J. Angus, Phil. Mag. 40 (1949) 929.

(b) G.D. Alkhazov, Nucl. Instr. and Meth. 89 (1970) 155 .

[19] M. Aderholz, P. Lazeyras, I. Lehraus, R. Matthewson and W. Tejessy, Nucl. Instr. and Meth. 118 (1974) 419.

[20] J.H. Carver and P. Mitchell, Nucl. Instr. and Meth. 52 (1967) 130.

[21] J. Byrne, Nucl. Instr. and Meth. 74 (1969) 291.

[22] T.E. Steward, G.S. Hurst, T.E. Bortner, J.E. Parks, F.W. Martin and H.L.R. Weidner, J. Opt. Soc. Am. 60 (1970) 1290.

[23] Rapport d'activité 1978-1979, LURE, CNRS-Université Paris Sud (1979).

[24] F. Sauli, Yellow Report CERN 79-09.

[25] U. Fano and J.W. Cooper, Rev. Mod. Phys. 40 (1968) 441.

[26] G.H. Lawrence, Phys. Rev. 175 (1968) 40.

[27] J.R. Samson, Adv. At. Molec. Phys. 2 (1968) 177.

[28] U. Fano, Ann. Rev. Nucl. Sci. 13 (1963) 1.

[29] J.D. Jackson, Classical electrodynamics (2nd ed.; J. Wiley, New York).

[30] G. Liggett and J.S. Levinger, J. Opt. Soc. Am. 58 (1968) 109.

[31] M.J. Van der Wiel and G. Wiebes, Physica 53 (1971) 225.

[32] At. Data Nucl. Data Tables 19 (1977) 100.

[33] C.B. Opal, E.C. Beaty and W.K. Peterson, At. data 4 (1972) 209.

[34] C.B. Opal, W.K. Peterson and E.C. Beaty, J. Chem. Phys. 55 (1971) 4100.

[35] J. Fryar and J.W. McConkey, J. Phys. B Atom. Mol. Phys. 9 (1976) 619.

[36] B. Grosswendt Ewaibel, Nucl. Instr. and Meth. 155 (1978) 145.

[37] B.L. Moiseiwitsch and S.J. Smith, Rev. Mod. Phys. 40 (1968) 238

[38] I.P. Zapesochnyi and P.V. Feltsan, Opt. Spectrosc. USSR 20 (1966) 291.

[39] H.S. Snyder, Phys. Rev. 72 (1947) 181. 